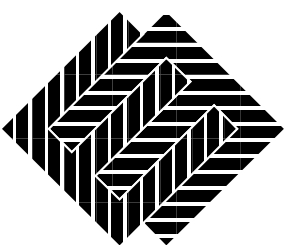

ENGINEERING MECHANICS

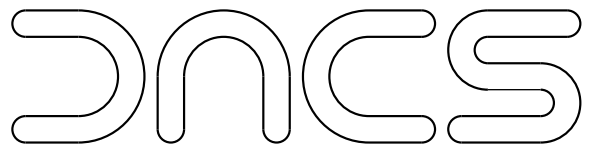

Delft Aerospace Computational Science

Space/Time Multigrid for a

Fluid-Structure-Interaction Problem

E.H. van Brummelen, K.G. van der Zee and R. de Borst

E.H.vanBrummelen@tudelft.nl

Report DACS-06-004

May 2006 
Copyright (C) 2006: The copyright of this manuscript resides with the author.

All rights reserved. This work may not be translated or copied

in whole or in part without the written permission of the author.

TUD/LR/EM

Kluyverweg 1, 2629HS Delft, The Netherlands

P.O. Box 5058, 2600 GB Delft, The Netherlands

Phone: +31 152785460 Fax: +31 152611465

ISSN 1574-6992 


\title{
SPace/Time Multigrid for a Fluid-Structure-Interaction Problem
}

\author{
E.H. van Brummelen, K.G. van der Zee and R. de Borst \\ Delft University of Technology, Faculty of Aerospace Engineering \\ P.O. Box 5058, 2600 GB Delft, The Netherlands
}

\begin{abstract}
The basic iterative method for solving fluid-structure-interaction problems is a defect-correction process based on a partitioning of the underlying operator into a fluid part and a structural part. In the present work we establish for a prototypical model problem that this defect-correction process yields an excellent smoother for multigrid, on account of the relative compactness of the fluid part of the operator with respect to the structural part. We show that the defect-correction process in fact represents an asymptotically-perfect smoother, i.e., the effectiveness of the smoother increases as the mesh is refined. Consequently, on sufficiently fine meshes the fluid-structure-interaction problem can be solved to arbitrary accuracy by one iteration of the defect-correction process followed by a coarse-grid correction. Another important property of the defect-correction process is that it smoothens the error in space/time, so that the coarsening in the multigrid method can be applied in both space and time.
\end{abstract}

2000 Mathematics Subject Classification: primary: 65N12. secondary: 35R35, 65N30

Keywords and Phrases: fluid-structure interaction, space/time multigrid, relatively-compact partitions, asymptotically-perfect smoothing, subiteration, space/time finite-element methods.

\section{Introduction}

Interactions between a flexible structure and a contiguous fluid flow are of critical importance in many engineering disciplines. Numerical solution procedures for such fluid-structure-interaction problems are confronted with two daunting challenges. First, the fluid and the structure typically display distinctly different length and time scales and, accordingly, the aggregated fluid-structure-interaction problem bears a pronounced multiscale character. Second, the interaction occurs at a free boundary, which yields a complicated interconnection between the governing initial-boundary-value problems, and the domains on which these are defined. In addition, several practical impediments emanate from the inherent interconnection between the fluid and structure, such as the loss of software modularity.

The customary approach to bypass these problems is through partitioning. The fluid and structure equations are then solved alternately subject to complementary partitions of the interface conditions. This iterative process, commonly referred to as subiteration, essentially corresponds to a defectcorrection process [2] based on a partition of the operator that underlies the fluid-structure-interaction problem into a structural part and a fluid part. The essential deficiency of partitioned methods pertains to their convergence behavior. Regularly, convergence is excessively slow, or prohibitively small time steps are required to maintain stability. Moreover, the error-amplification operator associated with the subiteration process can be nonnormal, which yields a severe degradation in the robustness and efficiency of the method; see [4].

In the present work we establish for a prototypical fluid-structure-interaction model problem, viz., the panel problem, that the defect-correction process corresponding to the subiteration method provides a suitable smoother for a multigrid process, in spite of its inadequacy as a solver. In the model problem, the action of the fluid on the structure is represented by a so-called displacement-to-pressure (dtp) operator, viz., an operator that associates to each admissible displacement of the structure the corresponding pressure exerted by the fluid on the structure. This dtp operator constitutes an inseparable nonlocal integro-differential operator in space/time. A pivotal element of the multigrid 
theory for the model problem is the relative compactness of the dtp operator with respect to the structural operator. More precisely, denoting by $A$ the aggregated fluid-structure-interaction operator and by $S+P=A$ its partition into the structural operator $S$ and the dtp operator $P$, it holds that $S$ is an isomorphism between an appropriate space/time Hilbert space $U$ and a dual space $V$, whereas $P$ maps $U$ into a space $Y^{\prime}$ with compact embedding in $V^{\prime}$. The embedding of $Y^{\prime}$ into $V^{\prime}$ forms a subspace of regular (smooth) functions in $V^{\prime}$. Conversely, $S^{-1}$ maps $Y^{\prime}$ into a space $X$ with compact embedding in $U$ and the embedding $X \hookrightarrow U$ forms a regular subspace of $U$. The error-amplification operator pertaining to the defect-correction process is $S^{-1} P$ and, hence, the defect-correction process regularizes (smoothens) the error. On account of its regularity, the smoothened error can be accurately represented on a coarse mesh. Hence, the fluid-structure-interaction problem can be solved effectively by means of subiteration enhanced with coarse-grid correction and, by recursion, by multigrid. It is noteworthy that the relative compactness can in fact be construed as the origin of the multiscale character of the fluid-structure-interaction problem. In this context, the multigrid method makes a virtue of necessity in engaging the multiscale character of fluid-structure-interaction problems.

The smoothing provided by the defect-correction process has the fundamental property that the error in the coarse-grid representation (projection) of the smoothened error vanishes as the mesh is refined. This implies that the effectiveness of the smoother improves as the mesh becomes finer. On sufficiently fine meshes, the fluid-structure-interaction problem can therefore be solved by one iteration of the defect-correction process followed by a coarse-grid correction. We refer to a smoothing process with this property as an asymptotically-perfect smoother. Another important property of the defectcorrection process is that it smoothens the error in space/time. Hence, the coarse-grid correction can be extracted from a mesh that is coarser in both space and time. This motivates us to endow the corresponding multigrid method with the predicate space/time multigrid.

The contents of this paper are organized as follows: Section 2 presents the integro-differential model problem. Moreover, this section establishes the appropriate functional setting for the model problem and the relative compactness of the dtp operator with respect to the structural operator in this functional setting. In section 3 we develop a variational theory of the multigrid method. This variational theory enables us to establish the connection between relatively-compact partitions of operators and the asymptotically-perfect-smoothing property of defect-correction processes based on such partitions. In section 4 we attend to the details for the model problem. In particular, we derive a regularity theorem that asserts that the inverse of the structural operator indeed maps regular compact subsets of the dual space into regular compact subsets of the primal space. Furthermore, we elaborate several practical aspects of the space/time multigrid process for the model problem. Numerical experiments and results are presented in section 5. Finally, section 6 contains concluding remarks.

\section{Problem statement}

In this paper we are concerned with the analysis of a space/time multigrid method for an integrodifferential model of a prototypical fluid-structure-interaction problem, viz., the panel problem. Below, we first specify the integro-differential model. The structural part of the operator corresponds to a separable differential operator. The fluid part is represented by the so-called displacement-to-pressure (dtp) operator, viz., an inseparable nonlocal operator in space/time. Next, we introduce the necessary definitions and notation and recall some classical results for the structure separately. To accommodate the inseparable dtp operator, we recast the model problem into a variational space/time formulation. Finally, we establish that the dtp operator is relatively compact with respect to the structural operator. This relative compactness of the dtp operator forms an essential element of the multigrid theory for the model problem.

2.1 Integro-differential model of the panel problem

To formulate the model problem, we consider an open bounded unit (spatial) interval $\Omega:=] 0,1[$, an open bounded (temporal) interval $] 0, T[$, and the (space/time) cylinder $Q:=\Omega \times] 0, T[$. The boundary 

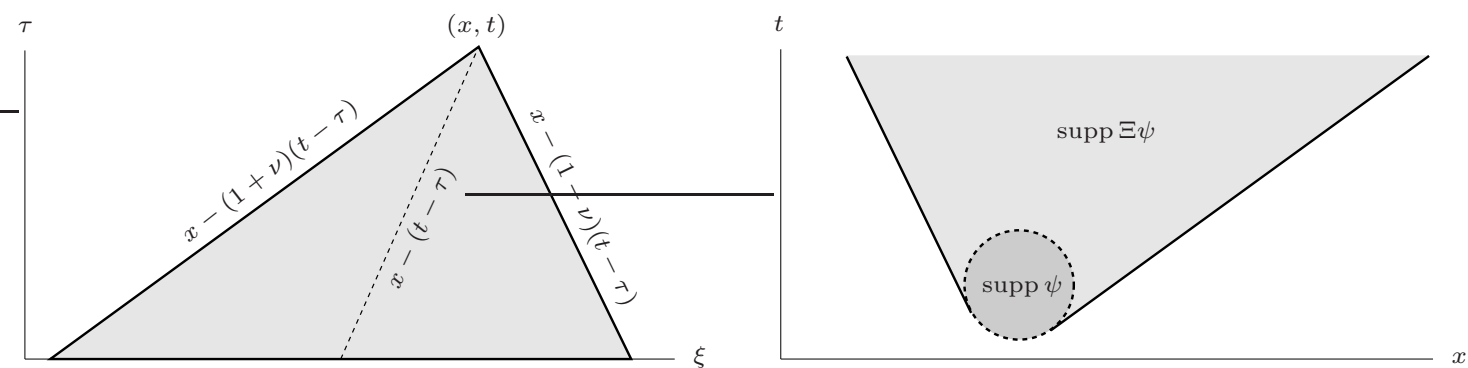

Figure 1: Illustration of the support of the kernel in the integral operator $\Xi$, viz., the set $(2.2)$ (left) and of the support of $\Xi \psi$ for a compactly supported function $\psi$ (right).

$\partial Q=$ closure $Q-Q$ can be separated into the initial and final temporal boundaries $\Gamma_{0}=\Omega \times\{0\}$ and $\Gamma_{T}:=\Omega \times\{T\}$ and the lateral boundary $\Gamma_{s}=\{0,1\} \times[0, T]$.

The integro-differential model of the panel problem is formally defined by the initial-boundaryvalue problem:

$$
S_{\lambda} u+\mu P_{\nu} u=0, \quad(x, t) \in Q,
$$

with $S_{\lambda}=(\cdot)^{\prime \prime}+\lambda^{2} D^{4},(\cdot)^{\prime}=\partial / \partial t$ and $D=\partial / \partial x$, subject to the homogeneous boundary conditions

$$
u(x, t)=0, \quad D u(x, t)=0, \quad(x, t) \in \Gamma_{s},
$$

and the initial conditions

$$
u(x, t)=\varphi_{0}(x), \quad u^{\prime}(x, t)=\varphi_{1}(x), \quad(x, t) \in \Gamma_{0},
$$

for certain prescribed functions $\varphi_{0}$ and $\varphi_{1}$ from $\Omega$ into $\mathbb{R}$. The integro-differential operator $P_{\nu}(\nu>0)$ can be factored into a product of differential and integral operators as

$$
P_{\nu}=\pi^{-1} \Psi \Xi \Psi
$$

where $\Psi(\cdot)=\nu D(\cdot)+(\cdot)^{\prime}$ and

$$
[\Xi \psi](x, t)=\int_{Q} \psi(\xi, \tau) \frac{H((t-\tau)-|(x-\xi)-\nu(t-\tau)|)}{\sqrt{(t-\tau)^{2}-|(x-\xi)-\nu(t-\tau)|^{2}}} d \xi d \tau,
$$

with $H(\cdot)$ the Heaviside function. The mapping $u \mapsto \mu P_{\nu} u$ associates with each admissible structure displacement $u: Q \rightarrow \mathbb{R}$ the corresponding pressure exerted on the structure by the inviscid compressible fluid. Accordingly, we refer to $P_{\nu}$ as the displacement-to-pressure (dtp) operator.

It is important to note that the integral operator (2.1e) renders the dtp operator nonlocal in space/time: for each $(x, t)$ the support of the kernel in $(2.1 \mathrm{e})$ consists of the set

$$
\left\{(\xi, \tau) \in \mathbb{R}^{2}: \tau<t, x-(1+\nu)(t-\tau)<\xi<x-(1-\nu)(t-\tau)\right\} ;
$$

see Figure 1 (left). The set (2.2) represents a triangle in space/time, delineated by the characteristics through $(x, t)$ of the wave equation that underlies the dtp operator. Conversely, considering a function $\psi$ with compact support, the support of $\Xi \psi$ consists of all $(x, t)$ for which $(2.2)$ intersects with supp $\psi$; see Figure 1 (right). Clearly, supp $\Xi \psi \not \subset \operatorname{supp} \psi$ and, hence, $\Xi$ is a nonlocal operator in space/time. Moreover, because the kernel in (2.1e) is inseparable in space/time, the dtp operator is inseparable in space/time.

The panel-model problem (2.1) and, in particular, the dtp operator can be found in several classical references on aeroelasticity, for instance, Refs. [1,7]. To enable an interpretation of (2.1), we mention here that the parameters $\lambda, \mu$ and $\nu$ in (2.1) characterize the stiffness of the structure, the fluid-tostructure mass ratio, and the Mach number of the fluid flow, respectively. 


\subsection{Definitions and notation}

The structure problem separately, i.e., problem (2.1a) with $\mu=0$, corresponds to an evolution equation of the second order in $t$ with an elliptic operator. A comprehensive general theory for such problems is presented in [12]. Therefore, we adhere mostly to the notational conventions in this reference. A synopsis is presented below.

Considering an arbitrary open set $\Omega \subseteq \mathbb{R}^{n}$, we denote by $H^{m}(\Omega)\left(m \in \mathbb{Z}_{+}=\{0,1,2, \ldots\}\right)$ the Sobolev space of functions on $\Omega$ which reside in $L^{2}$ together with their distributional partial derivatives of order $\leq m$. Equipped with the innerproduct $(\cdot, \cdot)_{H^{m}(\Omega)}$,

$$
(u, v)_{H^{m}(\Omega)}=\sum_{|\alpha| \leq m} \int_{\Omega} D^{\alpha} u(x) D^{\alpha} v(x) d x,
$$

with $\alpha \in \mathbb{Z}_{+}^{n}$ a multi-index, $|\alpha|=\alpha_{1}+\cdots+\alpha_{n}$ and $D^{\alpha}=\partial^{|\alpha|} / \partial x_{1}^{\alpha_{1}} \cdots \partial x_{n}^{\alpha_{n}}$ the distributional derivative of order $\alpha, H^{m}(\Omega)$ is a Hilbert space. The norm induced by the innerproduct $(2.3)$ is denoted by $\|\cdot\|_{H^{m}(\Omega)}$.

Let $\mathscr{D}(\Omega)$ represent the class of test functions on $\Omega$, viz., the infinitely differentiable functions with compact support in $\Omega$. The space of distributions, $\mathscr{D}^{\prime}(\Omega)$, is defined as the aggregate of all bounded linear functionals on $\mathscr{D}(\Omega)$. The space $H_{0}^{m}(\Omega)$, defined as the closure of $\mathscr{D}(\Omega)$ in the $H^{m}(\Omega)$ norm, may be identified with the subspace of $H^{m}(\Omega)$ consisting of functions that vanish on the boundary $\partial \Omega$ together with their distributional derivatives upto and including order $m-1$.

The dual of a Hilbert space $H$, i.e., the aggregate of all continuous linear functionals on $H$, is denoted by $H^{\prime}$. The duality pairing $\langle\cdot, \cdot\rangle$ is the functional on $H^{\prime} \times H$ defined by $\langle f, u\rangle:=f(u)$. Riesz' representation theorem asserts that there exists an isomorphism $\mathfrak{J}: H^{\prime} \mapsto H$ such that $\langle f, u\rangle=$ $(\mathfrak{J}(f), u)_{H}$ for all $u \in H$. By means of the ambiguous notation $f=\mathfrak{J}(f)$ we can identify $H$ with its dual. A Hilbert space $H$ endowed with the identification $H=H^{\prime}$ is called a pivot space. If $V$ is a closed subspace of the pivot space $H$, then $V^{\prime}$ can be identified with a superspace of $H$. The definition of the Sobolev spaces $H^{m}(\Omega)$ is extended to negative integers by the definition $H^{-m}(\Omega):=\left[H_{0}^{m}(\Omega)\right]^{\prime}$ $\left(m \in \mathbb{Z}_{+}\right)$, under the convention that $H^{0}(\Omega)\left(=L^{2}(\Omega)\right)$ acts as pivot space. For further elaboration, see Ref. [12].

If $H$ is a Hilbert space, then $L^{2}(a, b ; H)$ is the Bochner space of functions $u$ from $] a, b[$ into $H$ such that $\|u\|_{L^{2}(a, b ; H)}<\infty$ with

$$
\|u\|_{L^{2}(a, b ; H)}^{2}=(u, u)_{L^{2}(a, b ; H)}, \quad(u, v)_{L^{2}(a, b ; H)}=\int_{a}^{b}(u(t), v(t))_{H} d t,
$$

Endowed with the innerproduct in $(2.4), L^{2}(a, b ; H)$ is a Hilbert space. In addition, we define by $B(a, b ; H)$ the continuous functions from $[a, b]$ into $H$, which is a Banach space under the norm $\|\cdot\|_{B(a, b ; H)}=\sup _{t \in[a, b]}\|u(t)\|_{H}$.

\subsection{Classical results for the structure separately}

The following proposition follows immediately from the general theory in [12, Sec.3.8]:

Proposition 2.1. Given an ordered pair $\left(\varphi_{0}, \varphi_{1}\right) \in H_{0}^{2}(\Omega) \times L^{2}(\Omega)$, and a function $f \in$ $L^{2}\left(0, T ; L^{2}(\Omega)\right)$, there exists a unique function $u \in L^{2}\left(0, T ; H_{0}^{2}(\Omega)\right)$ with $u^{\prime} \in L^{2}\left(0, T ; L^{2}(\Omega)\right)$ such that

$$
u^{\prime \prime}(t)+\lambda^{2} D^{4} u(t)=f, \quad u(0)=\varphi_{0}, \quad u^{\prime}(0)=\varphi_{1} .
$$

Moreover, it holds that $u^{\prime \prime} \in L^{2}\left(0, T ; H^{-2}(\Omega)\right)$.

Hence, for $\mu=0$ it holds that problem (2.1) is well-posed in the functional setting specified by the premises of the the proposition. Note that $f=0$ for $(2.1)$.

It is important to note that there is some ambiguity in the enforcement of the initial conditions, because $u$ and $u^{\prime}$ are only set in an $L^{2}$ Bochner space. Therefore, a pointwise interpretation of $u$ and $u^{\prime}$ is not allowed. However, since $D^{4}: H_{0}^{2}(\Omega) \rightarrow H^{-2}(\Omega)$, equation (2.5) indeed implies that 
$u^{\prime \prime} \in L^{2}\left(0, T ; H^{-2}(\Omega)\right)$. The pointwise interpretation of $u$ and $u^{\prime}$ is then enabled by a remarkable result for interpolation spaces $[12, \S 2]$ : if $u \in W^{m}(a, b)$,

$$
W^{m}(a, b)=\left\{u \in L^{2}\left(a, b ; X_{0}\right): u^{(m)} \in L^{2}\left(a, b ;\left(X_{1}\right)\right\}\right.
$$

with $u^{(m)}=d^{m} u / d t^{m}$, then

$$
u^{(j)} \in B\left(a, b ;\left[X_{0}, X_{1}\right]_{(j+1 / 2) / m}\right), \quad 0 \leq j \leq m-1,
$$

where $\left[X_{0}, X_{1}\right]_{\theta}$ is the interpolation space between $X_{0}$ and $X_{1}$. The definition of the interpolation space is technical; see $[12, \S 2]$ for details. Specifically, however, for $0<\theta_{0}<\theta_{1}<1$ we have the inclusion relations

$$
X_{0}=\left[X_{0}, X_{1}\right]_{0} \subset\left[X_{0}, X_{1}\right]_{\theta_{0}} \subset\left[X_{0}, X_{1}\right]_{\theta_{1}} \subset\left[X_{0}, X_{1}\right]_{1}=X_{1} .
$$

Hence, with $u \in L^{2}\left(0, T ; H_{0}^{2}(\Omega)\right)$ and $u^{\prime \prime} \in L^{2}\left(0, T ; H^{-2}(\Omega)\right)$, both $u$ and $u^{\prime}$ represent continuous functions from $] 0, T$ [ into an interpolation space and, accordingly, they admit a pointwise interpretation in that interpolation space. Moreover, the initial conditions reside in proper subspaces of the respective interpolation spaces, so that the specification of the initial conditions is in fact appropriate.

\subsection{Variational space/time formulation}

The above exposition for $\mu=0$ relies on the separability of the structure operator $S_{\lambda}$ into a spatial and a temporal constituent. For $\mu>0$, however, such a separation is impeded by the inseparability of the dtp operator. More precisely, the dtp operator represents an inseparable nonlocal operator in space/time. As such, the dtp operator interferes in an essential manner with the classical theory set forth in section 2.3.

To bypass the complications posed by the introduction of the dtp operator in the classical setting, we first recast (2.5) and the corresponding functional setting into an equivalent variational space/time formulation. To this end, we recall the definition of the space/time cylinder $Q=\Omega \times] 0, T[$. We construct the space/time Hilbert space $\mathbb{H}(Q)$ in two steps. First, we define $\mathbb{H}(Q)$ as the collection of functions $u \in L^{2}(Q)$ such that the map $t \mapsto\left(u, u^{\prime}\right)(\cdot, t)$ resides in $L^{2}\left(0, T ; H_{0}^{2}(\Omega) \times L^{2}(\Omega)\right)$. Hence, $\mathbb{H}(Q)$ is the space/time equivalent of $\left\{u \in L^{2}\left(0, T ; H_{0}^{2}(\Omega)\right): u^{\prime} \in L^{2}\left(0, T ; L^{2}(\Omega)\right)\right\}$. We endow $\mathbb{H}(Q)$ with the inner product and norm

$$
(u, v)_{\mathbb{H}(Q)}=\left(u^{\prime}, v^{\prime}\right)_{L^{2}(Q)}+\left(D^{2} u, D^{2} v\right)_{L^{2}(Q)}, \quad\|u\|_{\mathbb{H}(Q)}^{2}=(u, u)_{\mathbb{H}(Q)}
$$

It follows immediately that $\mathbb{H}(Q)$ is a Hilbert under the inner product and norm provided by (2.9); cf. section 2.2. To facilitate the introduction of the initial conditions, we moreover define the spaces

$$
\mathbb{H}_{0}(Q)=\left\{u \in \mathbb{H}(Q):\left.u\right|_{\Gamma_{0}}=\varphi_{0}\right\}, \quad \mathbb{H}_{T}(Q)=\left\{u \in \mathbb{H}(Q):\left.u\right|_{\Gamma_{T}}=0\right\}
$$

With the above definitions, the structure problem can be condensed into the variational space/time formulation: find $u \in \mathbb{H}_{0}(Q)$ such that

$$
a_{0}(u, v)=b(v) \quad \forall v \in \mathbb{H}_{T}(Q),
$$

where the bilinear functional $a_{0}: \mathbb{H}_{0}(Q) \times \mathbb{H}_{T}(Q) \rightarrow \mathbb{R}$ and the linear functional $b: \mathbb{H}_{T}(Q) \rightarrow \mathbb{R}$ are defined by

$$
a_{0}(u, v)=\lambda^{2}\left(D^{2} v, D^{2} u\right)_{L^{2}(Q)}-\left(v^{\prime}, u^{\prime}\right)_{L^{2}(Q)}, \quad b(v)=\left(\varphi_{1},\left.v\right|_{\Gamma_{0}}\right)_{L^{2}(\Omega)} .
$$

In (2.11), the initial condition $\left.u^{\prime}\right|_{\Gamma_{0}}=\varphi_{1}$ is weakly enforced. Let us remark that alternative formulations are possible, e.g., by also enforcing the initial condition $\left.u\right|_{\Gamma_{0}}=\varphi_{0}$ weakly based on the 
transposition formalism; see $[12, \S 9]$. Well-posedness of $(2.11)$ can be established by means of the generalized Lax-Milgram (or BNB) theorem; see, for instance, $[8,16]$. However, it is an almost immediate consequence of proposition (2.1) and therefore we will not further pursue it here. Conversely, because the generalized Lax-Milgram theorem is a necessary condition for well-posedness (see [8]), it holds that the bilinear form $a_{0}(\cdot, \cdot)$ is bounded and weakly coercive on $\mathbb{H}_{0}(Q) \times \mathbb{H}_{T}(Q)$.

The essential advantage of the above space/time formulation is that it provides a setting in which the dtp operator can be directly introduced. The dtp operator can be associated with a bilinear functional $p_{\nu}: \mathbb{H}_{0}(Q) \times \mathbb{H}_{T}(Q) \rightarrow \mathbb{R}$ according to $p_{\nu}(u, v)=\left(P_{\nu} u, v\right)_{L^{2}(Q)}$ and the initial-boundaryvalue-problem (2.1) can be condensed into the variational form: find $u \in \mathbb{H}_{0}(Q)$ such that

$$
a_{\mu}(u, v)=0 \quad \forall v \in \mathbb{H}_{T}(Q),
$$

where $a_{\mu}(u, v)=a_{0}(u, v)+\mu p_{\nu}(u, v)$. More precisely, the space/time setting allows us to conceive of $P_{\nu}$ as a perturbation of $S_{\lambda}$. In section 2.5 we shall establish that $P_{\nu}$ is a compact operator from $\mathbb{H}_{0}(Q)$ into $\mathbb{H}_{T}^{\prime}(Q)$, so that the Fredholm alternative holds; see, e.g., [13, theorem 2.27] or [20, §5.2]. In particular, this implies that (2.12) is well-posed, except for countably many isolated values of $1 / \mu$ corresponding to the eigenvalues of the operator $-S_{\lambda}^{-1} P_{\nu}$; see also [11, §3.6.7]. For such values of $\mu$ the null-space of $S_{\lambda}+\mu P_{\nu}$ is finite dimensional.

\subsection{Compactness of the dtp operator}

A fundamental element of the multigrid theory for problem (2.1) pertains to the compactness of the dtp operator. The structure operator $S_{\lambda}$ is an isomorphism from $\mathbb{H}_{0}(Q)$ to $\mathbb{H}_{T}^{\prime}(Q)$. On the other hand, the range of $P_{\nu}$ is a compact subspace of $\mathbb{H}_{T}^{\prime}(Q)$, i.e., $P_{\nu}$ is compact as an operator from $\mathbb{H}_{0}(Q)$ into $\mathbb{H}_{T}^{\prime}(Q)$.

We shall establish the compactness of $P_{\nu}$ by means of the Fourier transform:

$$
[F u](\kappa, \omega)=\hat{u}(\kappa, \omega)=\frac{1}{2 \pi} \int_{\mathbb{R}^{2}} \mathrm{e}^{-i(\kappa x+\omega t)} u(x, t) d x d t .
$$

Here we assume that $u$ is appropriately extended outside $Q$ so that it is well-defined on $\mathbb{R}^{2}$. We recall that the Fourier transform (2.13) provides an isomorphism of $L^{2}\left(\mathbb{R}^{2}\right)$ onto $L^{2}\left(\mathbb{R}^{2}\right)$ with inverse

$$
\left[F^{-1} \hat{u}\right](x, t)=\frac{1}{2 \pi} \int_{\mathbb{R}^{2}} \mathrm{e}^{i(\kappa x+\omega t)} \hat{u}(\kappa, \omega) d \kappa d \omega .
$$

Throughout, we shall suppress the nuisance factors, viz., the powers of $(2 \pi)^{-1}$.

Let us consider the image of a function $\psi \in L^{2}\left(\mathbb{R}^{2}\right)$, specified by its Fourier transform, under the integral operator $\Xi$ according to (2.1e). Upon restricting the integration interval in $(\xi, \tau)$ to account for the support of the kernel in (2.1e) and suitably rearranging the order of integration, we obtain:

$$
[\Xi \psi](x, t)=\int_{\mathbb{R}^{2}} \mathrm{e}^{i(\kappa x+\omega t)} \hat{\Xi}(\kappa, \omega, x, t) \hat{\psi}(\kappa, \omega) d \kappa d \omega,
$$

where the Fourier-symbol of $\Xi$ is given by

$$
\hat{\Xi}(\kappa, \omega, x, t)=\int_{0}^{t} \int_{x-(\nu+1)(t-\tau)}^{x-(\nu-1)(t-\tau)} \frac{\mathrm{e}^{-i(\kappa(x-\xi)+\omega(t-\tau))}}{\sqrt{(t-\tau)^{2}-|(x-\xi)-\nu(t-\tau)|^{2}}} d \xi d \tau .
$$

Introducing the transformation $(\theta, \eta) \mapsto(\xi, \tau)=(x-(\nu-\sin \theta) \eta, t-\eta)$ into (2.15b) and applying the partition of unity $1=\cos ^{2} \theta+\sin ^{2} \theta$, we obtain

$$
\hat{\Xi}(\kappa, \omega, x, t)=-\int_{-\pi / 2}^{\pi / 2} \int_{0}^{t} \mathrm{e}^{-i(\kappa(\nu-\sin \theta)+\omega) \eta} d \eta d \theta=i \int_{-\pi / 2}^{\pi / 2} \frac{1-\mathrm{e}^{-i(\kappa(\nu-\sin \theta)+\omega) t}}{\kappa(\nu-\sin \theta)+\omega} d \theta .
$$


Let us note that (2.16) conveys that $\hat{\Xi}(\cdot)$ is in fact independent of $x$. Equation (2.16) leads us to the upper bound:

$$
|\hat{\Xi}(\kappa, \omega, x, t)|^{2} \lesssim\left(1+\kappa^{2}+\omega^{2}\right)^{-1 / 2} \quad \forall(\kappa, \omega) \in \mathbb{R}^{2},
$$

where the binary relation $\lesssim$ indicates that the left member is at most equal to the right member times a bounded constant. The derivation of the majorization (2.17) is presented in Appendix A. Formally defining $H^{s}(Q)\left(s \in \mathbb{R}_{+}\right)$as the aggregate of all functions in $L^{2}(Q)$ that are bounded in the norm $\|\cdot\|_{s}$,

$$
\|u\|_{s}^{2}=\int_{\mathbb{R}^{2}}\left(1+\kappa^{2}+\omega^{2}\right)^{s}|\hat{u}(\kappa, \omega)|^{2} d \kappa d \omega
$$

it follows from (2.17) that $\Xi: L^{2}(Q) \rightarrow H^{1 / 2}(Q)$. Let us mention that for $s \in \mathbb{Z}_{+}$the norm defined in (2.18) is equivalent to $\|\cdot\|_{H^{s}(Q)}$, so that the above definition of $H^{s}$ can be regarded as an extension of the Sobolev spaces to non-integer orders. For negative non-integer orders $H^{-s}(Q)$ can again be identified with the dual of $H^{s}(Q)$ with $L^{2}(Q)$ acting as the pivot space. It is easily established that $\Psi: H^{s}(Q) \rightarrow H^{s-1}(Q)$ and, hence, $P_{\nu}: H^{s}(Q) \rightarrow H^{s-3 / 2}(Q)$. In summary, $P_{\nu}: \mathbb{H}_{0}(Q) \rightarrow H^{-1 / 2}(Q)$ on account of the straightforward inclusion $\mathbb{H}(Q) \subset H^{1}(Q)$ and the following sequence of injections:

$$
H^{1}(Q) \stackrel{\Psi}{\longrightarrow} L^{2}(Q) \stackrel{\Xi}{\longrightarrow} H^{1 / 2}(Q) \stackrel{\Psi}{\longrightarrow} H^{-1 / 2}(Q) .
$$

Moreover, the inclusion $\mathbb{H}_{T}(Q) \subset H^{1}(Q)$ implies $\mathbb{H}_{T}^{\prime}(Q) \supset H^{-1}(Q)$. From the compactness of the embedding $H^{s+\epsilon}(Q) \hookrightarrow H^{s}(Q)$ for $\epsilon>0$ (see, for instance, [19, p.1026 ff.]) it then follows that the embedding of the range of $P_{\nu}$ with domain $\mathbb{H}_{0}(Q)$ into $\mathbb{H}_{T}^{\prime}(Q)$ is compact, i.e., $P_{\nu}$ is a compact operator from $\mathbb{H}_{0}(Q)$ into $\mathbb{H}_{T}^{\prime}(Q)$. Considering that the range of $S_{\lambda}$ coincides with $\mathbb{H}_{T}^{\prime}(Q)$, in terms of the operators $S_{\lambda}$ and $P_{\nu}$ this means that $P_{\nu}$ is relatively compact with respect to $S_{\lambda}$; see [11].

\section{Variational multigrid theory}

In this section we are concerned with the development of a variational theory of the multigrid methodology. Based on the two-grid method, section 3.1 elaborates the generic elements of the multigrid method, viz., the smoothing operation and the coarse-grid correction. In section 3.2 we establish for a canonical variational problem that if the underlying operator admits a partitioning into a regular and a relatively-compact constituent, then the defect-correction process based on this partitioning potentially provides a perfect smoother on sufficiently fine meshes. We refer to a smoothing process with this property as an asymptotically-perfect smoother. Finally, in section 3.3 we elaborate the asymptotically-perfect-smoothing property for piecewise-polynomial approximations in Sobolev spaces.

\subsection{Two-grid method}

Let $Q$ be an open bounded subset of $\mathbb{R}^{n}$, and let $U$ and $V$ be Hilbert spaces on $Q$ with inner products $(\cdot, \cdot)_{U}$ and $(\cdot, \cdot)_{V}$, respectively. Consider the canonical variational problem: find $u \in U$ such that

$$
a(u, v)=b(v) \quad \forall v \in V,
$$

where $a: U \times V \rightarrow \mathbb{R}$ is a bounded bilinear form and $b: V \mapsto \mathbb{R}$ is a bounded linear functional. We associate the bilinear and linear form with an operator $A: U \rightarrow V^{\prime}$ and a vector $b \in V^{\prime}$, respectively:

$$
a(u, v)=(A u, v)_{L^{2}(Q)}, \quad b(v)=(b, v)_{L^{2}(Q)} .
$$

Furthermore, we assume that the bilinear form $a(\cdot, \cdot)$ satisfies the conditions of the generalized LaxMilgram theorem, so that (3.1) admits a unique solution.

In conjunction with (3.1), we consider a sequence of (finite-element) approximation problems. Let $\left\{U_{l}\right\}$ and $\left\{V_{l}\right\}$ represent sequences of asymptotically dense nested subsets, i.e., $X_{0} \subset X_{1} \subset \cdots \subseteq X$ 
and $X_{l} \rightarrow X$ as $l \rightarrow \infty(X$ is $U$ or $V)$. Correspondingly, we obtain a sequence of approximation problems: find $u \in U_{l}$ such that

$$
a(u, v)=b(v) \quad \forall v \in V_{l},
$$

We assume that $a(\cdot, \cdot)$ complies with the hypotheses of the generalized Lax-Milgram theorem on $U_{l} \times V_{l}$ for all $l$, so that each approximation problem is well posed.

The two-grid method for the approximation problem (3.3) with index $l$ consists of two operations, viz., the smoothing operation and the coarse-grid-correction operation. The purpose of the smoothing operation is to reduce the component of the error in the orthogonal complement $U_{l-1}^{\perp}$ of $U_{l-1}$ in $U_{l}$. The coarse-grid correction, on the other hand, reduces the error component in $U_{l-1}$. To elucidate the two-grid method, let us rewrite the approximation problem with index $l$ in the multiscale form [10]: find $(\underline{u}, \check{u}) \in U_{l-1} \times U_{l-1}^{\perp}$ such that, simultaneously,

$$
\begin{array}{ll}
a(\underline{u}, \underline{v})+a(\check{u}, \underline{v})=b(\underline{v}) & \forall \underline{v} \in V_{l-1}, \\
a(\underline{u}, \check{v})+a(\check{u}, \check{v})=b(\check{v}) & \forall \check{v} \in V_{l-1}^{\perp} .
\end{array}
$$

If the (fine-scale) component $\check{u} \in U_{l-1}^{\perp}$ is given, then the complementary (coarse-scale) component $\underline{u} \in U_{l-1}$ can be extracted from (3.4a) and the solution to the approximation problem can be composed as $u=\underline{u}+\check{u}$. Let us denote by $\Pi_{U_{l-1}}: U \rightarrow U_{l-1}$ the orthogonal projection onto $U_{l-1}$ defined by

$$
\left(v, \Pi_{U_{l-1}} u\right)_{U}=(v, u)_{U} \quad \forall v \in U_{l-1} .
$$

The projection onto the orthogonal complement of $U_{l-1}$ in $U$ is then induced by the operator $I-\Pi_{U_{l-1}}$ with $I$ the identity in $U$. The fine-scale component $\check{u}$ can be identified as the projection of the actual solution $u$ of the approximation problem with index $l$ onto the orthogonal complement, i.e., $\check{u}=u-\Pi_{U_{l-1}} u$. The objective of the smoothing operation is to provide an approximation $\tilde{u} \in U_{l}$ such that its projection onto the orthogonal complement, $\tilde{u}-\Pi_{U_{l-1}} \tilde{u}$, is close to the projection of the actual solution, $\check{u}$. Upon replacing $\check{u}$ in $(3.4 \mathrm{a})$ by the approximation $\tilde{u}-\Pi_{U_{l-1}} \tilde{u}$, we obtain the coarse-scale problem: find $\tilde{u} \in U_{l-1}$ such that

$$
a(\underline{\tilde{u}}, \underline{v})=a\left(\Pi_{U_{l-1}} \tilde{u}, \underline{v}\right)+b(\underline{v})-a(\tilde{u}, \underline{v}) \quad \forall \underline{v} \in \tilde{V}_{l} .
$$

We note that the coarse-scale problem can be expressed in operator form as: find $\tilde{u} \in U_{l-1}$ such that

$$
(A \underline{\tilde{u}}, \underline{v})_{L^{2}(Q)}=\left(A \Pi_{U_{l-1}} \tilde{u}, \underline{v}\right)_{L^{2}(Q)}+(b-A \tilde{u}, \underline{v})_{L^{2}(Q)} \quad \forall \underline{v} \in V_{l-1} .
$$

Equation (3.7) conveys that (3.6) is in fact the variational form of the coarse-grid equation in the FullApproximation-Scheme; see $[3, \S 8]$. The coarse-scale solution $\tilde{u}$ is used to correct the approximation $\tilde{u} \in U_{l}$ according to $\tilde{u}+\tilde{u}-\Pi_{U_{l-1}} \tilde{u}$.

To analyze the convergence of the two-grid method, let us consider the approximation problem with index $l$ and let $\tilde{u}_{0} \in U_{l}$ represent a prescribed initial approximation. The error in $\tilde{u}_{0}$ is indicated by $e=\tilde{u}_{0}-u$, where $u \in U_{l}$ denotes the actual solution of the approximation problem. We represent the smoothing operation by the error-amplification operator $E: U_{l} \rightarrow U_{l}$, i.e., the post-smoothing error is represented by $E e$. By adding a suitable partition of zero to the coarse-scale equation (3.6), it follows that the error $E e$ in the post-smoothing approximation $\tilde{u}$ induces a coarse-scale error $\underline{e}=\underline{\tilde{u}}-\underline{u} \in U_{l-1}$ according to

$$
a(\underline{e}, \underline{v})=-a\left(\left(I-\Pi_{U_{l-1}}\right) E e, \underline{v}\right) \quad \forall \underline{v} \in V_{l-1} .
$$

Under the standing hypothesis that $a(\cdot, \cdot)$ complies with the premises of the generalized Lax-Milgram theorem on $U_{l} \times V_{l}$, equation (3.8) implies that $\|\underline{e}\|_{U} \lesssim\left\|\left(I-\Pi_{U_{l-1}}\right) E e\right\|_{U}$. This leads to the following upper bound for the error in the approximation $\tilde{u}+\underline{\tilde{u}}-\Pi_{U_{l-1}} \tilde{u}$ generated by the two-grid method:

$$
\begin{array}{r}
\left.\left\|\left(\tilde{u}+\tilde{u}-\Pi_{U_{l-1}} \tilde{u}\right)-u\right\|_{U}=\left\|\left(I-\Pi_{U_{l-1}}\right)(\tilde{u}-u)+(\underline{\tilde{u}}-\underline{u})\right\|_{U}=\|\left(I-\Pi_{U_{l-1}}\right) E e+\underline{e}\right) \|_{U} \\
\leq\left\|\left(I-\Pi_{U_{l-1}}\right) E e\right\|_{U}+\|\underline{e}\|_{U} \lesssim\left\|\left(I-\Pi_{U_{l-1}}\right) E e\right\|_{U} \leq\left\|\left(I-\Pi_{U_{l-1}}\right) E\right\|_{U}\|e\|_{U} .
\end{array}
$$


We note that the first identity in (3.9) follows by adding the partition of zero $\underline{u}-\Pi_{U_{l-1}} u$. The sequence of bounds (3.9) conveys that the convergence of the two-grid method is determined by the $\|\cdot\|_{U}$-norm of the operator $\left(I-\Pi_{U_{l-1}}\right) E$.

\subsection{Relatively-compact partitions and asymptotically-perfect smoothing}

We proceed under the assumption that the underlying operator $A$ in (3.1) admits a partitioning $A:=S+\mu P$ such that $P$ is relatively compact with respect to $S$. Moreover, we assume that the bilinear form $a_{0}(u, v)=(S u, v)_{L^{2}(Q)}$ satisfies the conditions of the generalized Lax-Milgram theorem, i.e., that $S$ possesses a bounded inverse $S^{-1}: V^{\prime} \rightarrow U$. It is to be noted that this setting encompasses the fluid-structure-interaction model problem (2.12).

Let us now consider a sequence of approximations $\left\{\tilde{u}_{i}\right\} \subset U$ generated by the following defectcorrection process [2], based on the partition of $A$ : given an initial approximation $\tilde{u}_{0} \in U$,

$$
a_{0}\left(\tilde{u}_{i+1}, v\right)=a_{0}\left(\tilde{u}_{i}, v\right)+b(v)-a_{\mu}\left(\tilde{u}_{i}, v\right) \quad \forall v \in V \quad(i=0,1,2, \ldots) .
$$

We denote by $e_{i}=\tilde{u}_{i}-u$ the error in the $i$-th approximation. By adding a suitable partition of zero to (3.10) and replacing the bilinear forms by their operator form for transparency, we obtain

$$
\left(S e_{i+1}, v\right)_{L^{2}(Q)}=\left(S e_{i}, v\right)_{L^{2}(Q)}-\left((S+\mu P) e_{i}, v\right)_{L^{2}(Q)} \quad \forall v \in V .
$$

As $S: U \rightarrow V^{\prime}$ is invertible under the standing hypotheses, it follows from (3.11) that $e_{i+1}=E e_{i}$ with $E=-\mu S^{-1} P$ the error-amplification operator of the defect-correction process.

On account of the relative compactness of $P$ with respect to $S$, the error-amplification operator possesses the essential property that it is compact from $U$ into $U$. To corroborate this assertion, let $Y^{\prime}$ represent the range of $P$ with domain $U$. On account of the compactness of the embedding $Y^{\prime} \hookrightarrow V^{\prime}$ and the closedness of $S$, the inverse $S^{-1}$ maps $Y^{\prime}$ into a subset $X$ with compact embedding in $U$. The compactness of the embedding $X \hookrightarrow U$ implies that $X$ can be equipped with a norm $\|\cdot\|_{X}$ that is stronger than $\|\cdot\|_{U}$ in the sense that for all $e \in X$ it holds that $\|e\|_{U} \lesssim\|e\|_{X}$. To define such a norm, let us note that $E$ admits a singular-value decomposition by virtue of its compactness; see [11, $\S 5.2 .3]$. Moreover, the singular-value decomposition can be used to construct a right inverse $E^{-1}$ :

$$
E(\cdot)=\sum_{i} \sigma_{i} g_{i}\left(h_{i}, \cdot\right)_{U}, \quad E^{-1}(\cdot)=\sum_{i} \sigma_{i}^{-1} h_{i}\left(g_{i}, \cdot\right)_{U},
$$

where $\left\{g_{i}\right\}$ and $\left\{h_{i}\right\}$ are orthonormal families in $U$ and $\left\{g_{i}\right\}$ is complete in $X$. The singular values $\left\{\sigma_{i}\right\}$ are repeated for multiplicity exceeding one and indexed in decreasing order, i.e, $\sigma_{0} \geq \sigma_{1} \geq \ldots>0$. One easily infers that $E E^{-1}$ indeed yields the identity on $X$. By means of (3.12) we can define the norm $\|\cdot\|_{X}=\left\|E^{-1}(\cdot)\right\|_{U}$. On account of the orthonormality of $\left\{g_{i}\right\}$ and $\left\{h_{i}\right\}$ and the completeness of $\left\{g_{i}\right\}$ in $X$, it holds that

$$
\begin{aligned}
\|e\|_{X}^{2}=\left\|E^{-1} e\right\|_{U}^{2}=\left(E^{-1} e, E^{-1} e\right)_{U}= & \sum_{i j}\left(\sigma_{i} \sigma_{j}\right)^{-1}\left(g_{i}, e\right)_{U}\left(g_{j}, e\right)_{U}\left(h_{i}, h_{j}\right)_{U} \\
& =\sum_{i} \sigma_{i}^{-2}\left(g_{i}, e\right)_{U}^{2} \geq \sigma_{0}^{-2} \sum_{i}\left(g_{i}, e\right)_{U}^{2}=\sigma_{0}^{-2}\|e\|_{U}^{2}
\end{aligned}
$$

Hence, $\|e\|_{U} \leq \sigma_{0}\|e\|_{X}$ and, as $\sigma_{0}$ is bounded by virtue of the boundedness of $E$, indeed $\|e\|_{U} \lesssim\|e\|_{X}$.

We refer to the defect-correction process (3.10) as an asymptotically-perfect smoother in conjunction with the approximation-space sequence $\left\{U_{l}\right\}$ if for all $\delta>0$ there exists an index $l(\delta)$ such that

$$
\sup _{e \in X} \frac{\left\|\left(I-\Pi_{U_{l(\delta)}}\right) e\right\|_{U}}{\|e\|_{X}}<\delta .
$$

Of course, as the approximation spaces in the sequence $\left\{U_{l}\right\}$ are nested, the bound (3.14) in fact holds for all $U_{l}$ with $l \geq l(\delta)$. It is important to note that the numerator and the denominator in (3.14) 
pertain to different norms. The expression (3.14) admits a convenient interpretation in terms of the interpolation error: for all $e \in X$ there exists an approximation space $U_{l(\delta)}$ such that the (relatively weak) $\|\cdot\|_{U}$-norm of the interpolation error $\left(I-\Pi_{U_{l}(\delta)}\right) e$ is bounded by $\delta$. In section 3.3 we exemplify (3.14) for Sobolev spaces and the usual piecewise-polynomial-approximation spaces associated with finite elements. To clarify the essence of (3.14) in the two-grid method, we recall that the upper bound (3.9) implies that the convergence of the two-grid method is determined by $\left\|\left(I-\Pi_{U_{l-1}}\right) E\right\|_{U}$. We then note that

$$
\left\|\left(I-\Pi_{U_{l}}\right) E\right\|_{U}=\sup _{e \in U} \frac{\left\|\left(I-\Pi_{U_{l}}\right) E e\right\|_{U}}{\|e\|_{U}}=\sup _{e \in X} \frac{\left\|\left(I-\Pi_{U_{l}}\right) e\right\|_{U}}{\left\|E^{-1} e\right\|_{U}}=\sup _{e \in X} \frac{\left\|\left(I-\Pi_{U_{l}}\right) e\right\|_{U}}{\|e\|_{X}} .
$$

Therefore, the condition (3.14) implies that on sufficiently fine approximation spaces the two-grid method consisting of a smoothing procedure based on one iteration of the defect-correction process (3.10) followed by the coarse-grid correction defined by (3.6) renders the error arbitrarily small. This motivates the predicate asymptotically-perfect smoother for the defect-correction process.

In specific cases it is generally not necessary to proceed through the inverse $E^{-1}$. If an interpolationerror estimate of the form $\left\|e-\Pi_{U_{l}} e\right\|_{U} \leq C\left(U_{l}\right)\|e\|_{X}(\forall e \in X)$ is available, then the following upper bound can be derived:

$$
\sup _{e \in U} \frac{\left\|\left(I-\Pi_{U_{l(\delta)}}\right) E e\right\|_{U}}{\|e\|_{U}} \leq C\left(U_{l(\delta)}\right) \sup _{e \in U} \frac{\|E e\|_{X}}{\|e\|_{U}}=C\left(U_{l(\delta)}\right)\|E\|_{\mathscr{L}(U, X)},
$$

where the operator norm $\|\cdot\|_{\mathscr{L}(U, X)}$ is defined by $\|E\|_{\mathscr{L}(U, X)}=\sup _{e \in U}\|E e\|_{X}\|e\|_{U}^{-1}$. Condition (3.14) can then be verified immediately on the basis of $C\left(U_{l}\right)$; see also section 3.3.

To facilitate the presentation, in the above exposition we have tacitly considered the original variational problem (3.1) instead of the approximation problems (3.3). However, the analysis extends immediately to the approximation problems. In particular, condition (3.14) is then replaced by

$$
\sup _{e \in U_{l(\delta)+1} \cap X} \frac{\left\|\left(I-\Pi_{U_{l(\delta)}}\right) e\right\|_{U}}{\|e\|_{X}}<\delta .
$$

The supremum being taken over the subset $U_{l(\delta)+1} \cap X$ instead of $X$, condition (3.17) is weaker than (3.14). The connotation of (3.17) is that the smoothing process need not reduce all the error components in $X$ that cannot be represented on $U_{l(\delta)}$, but just the ones that are present in the next-finer approximation space $U_{l(\delta)+1}$.

As a closing remark for this section, we mention that the relative compactness of $P$ in the partitioning $A=S+\mu P$ generally means that $S$ is the principal part of the operator $A$. Hence, the inverse $S^{-1}$ is a perfect smoother for the principal part. The above exposition thus confirms that on sufficiently fine meshes a good smoother for the principal part of an operator represents a good smoother for the operator itself; see [3].

\subsection{Piecewise-polynomial approximations in Sobolev spaces}

To illustrate the condition (3.14) pertaining to the asymptotically-perfect-smoothing property of the defect-correction process, let us consider the particular case that $U=H^{s}(Q)\left(s \in \mathbb{Z}_{+}\right)$and $X=$ $H^{s+\epsilon}(Q)(\epsilon>0)$. Moreover, we are concerned with finite-element-approximation spaces $U_{l}$ based on piecewise-polynomial functions. To define the finite-element-approximation spaces, let $\left\{\boldsymbol{Q}_{l}\right\}$ represent a nested sequence of partitions of $Q$, i.e., $\boldsymbol{Q}_{l}$ is a collection of non-overlapping open subdomains $Q_{e} \subset Q$ such that $\cup_{Q_{e} \in \boldsymbol{Q}_{l}}$ closure $Q_{e}=$ closure $Q$ and for each $Q_{e} \in \boldsymbol{Q}_{l+1}$ there exists a $\bar{Q}_{e} \in \boldsymbol{Q}_{l}$ such that $Q_{e} \subset \bar{Q}_{e}$. Furthermore, let $\left\{d_{l}\right\} \subset \mathbb{Z}_{+}$represent a nondecreasing sequence of polynomial degrees. The approximation spaces are specified as

$$
U_{l}=\mathscr{P}\left(\boldsymbol{Q}_{l}, d_{l}\right)=\left\{u \in H^{s}(Q):\left.u\right|_{Q_{e}} \text { is polynomial of degree } d_{l}\right\} \text {. }
$$


For simplicity we have assumed in (3.18) that the polynomial degree is uniform on the partitions $\boldsymbol{Q}_{l}$, but this assumption is nonessential. One can infer that indeed $U_{l} \subset U_{l+1}$.

Elementary interpolation theory in Sobolev spaces conveys that for all $e \in H^{r}(Q)$,

$$
\left\|e-\Pi_{U_{l}} e\right\|_{H^{s}(Q)} \lesssim h^{\varrho}\|e\|_{H^{r}(Q)},
$$

with $\varrho=\min \left\{d_{l}+1-s, r-s\right\}$ and $h$ the maximum of the diameters of $Q_{e} \in \boldsymbol{Q}_{l}$; see $[16, \S 8.5]$. In particular, with $E: U=H^{s}(Q) \rightarrow X=H^{s+\epsilon}(Q)$ this implies that

$$
\sup _{e \in H^{s}(Q)} \frac{\left\|\left(I-\Pi_{U_{l}}\right) E e\right\|_{H^{s}(Q)}}{\|e\|_{H^{s}(Q)}} \lesssim h^{\varrho} \sup _{e \in H^{s}(Q)} \frac{\|E e\|_{H^{s+\epsilon}(Q)}}{\|e\|_{H^{s}(Q)}}=h^{\varrho}\|E\|_{\mathscr{L}\left(H^{s}(Q), H^{s+\epsilon}(Q)\right)} \lesssim h^{\varrho},
$$

with $\varrho=\min \left\{d_{l}+1-s, \epsilon\right\}$. The first bound in (3.20) results from the upper bound on the interpolation error for $E e \in H^{s+\epsilon}(Q)$ provided by (3.19). The second bound follows from the boundedness of the operator $E: H^{s}(Q) \rightarrow H^{s+\epsilon}(Q)$. Assuming that $d_{l}+1-s>0$ and $h \rightarrow 0$ as $l \rightarrow \infty$, it holds that $h^{\varrho} \rightarrow 0$ as $l \rightarrow \infty$. Hence, the defect-correction process (3.10) is an asymptotically-perfect smoother in this setting.

\section{Space/time multigrid for the panel model}

This section is concerned with the application of the multigrid theory in section 3 to the fluid-structureinteraction model problem (2.12). The relative compactness of the dtp operator provides a necessary condition for the asymptotically-perfect smoothing property of (3.10) for the model problem. In section 4.1 we establish a regularity theorem for the structural operator that provides sufficiency. In section 4.2 we address several details of the multigrid method for the fluid-structure-interaction model problem. An important aspect is that (3.10) represents a space/time smoother for the model problem, so that the coarse-grid correction can be extracted from a mesh that is coarsened in both space and time.

\subsection{Interior regularity of the post-smoothing error}

The missing link that prevents us from applying the theory in section 3 to finite-element discretizations of (2.12) is a regularity theorem that precisely specifies the regularity of the solution $e \in \mathbb{H}_{0}(Q)$ of

$$
\left(S_{\lambda} e, v\right)_{L^{2}(Q)}=(b, v)_{L^{2}(Q)} \quad \forall v \in \mathbb{H}_{T}(Q),
$$

if $b$ resides in a regular subspace of $\mathbb{H}_{T}^{\prime}(Q)$. It is to be noted that by assumption the approximations comply with the initial conditions and, hence, the error $e$ resides in fact in $\mathbb{H}_{0}(Q)$ with homogeneous initial conditions. To elaborate the importance of (4.1), let us recall that the range of the dtp operator $P_{\nu}$ is a compact (regular) subspace of $\mathbb{H}_{T}^{\prime}(Q)$. Therefore, we are interested in the regularity of $e$ subordinate to (4.1) if $b$ resides in the range of $P_{\nu}$.

To enable the derivation of a sharp estimate, we introduce the norm:

$$
\|u\|_{s}^{2}=\int_{\mathbb{R}^{2}}\left(1+\kappa^{4}+\omega^{2}\right)^{s}|\hat{u}(\kappa, \omega)|^{2} d \kappa d \omega .
$$

To establish that the norm $\|\cdot\|_{1}$ is equivalent to $\|\cdot\|_{\mathbb{H}(Q)}$ for functions in $\mathbb{H}_{0}(Q)$, we note that $\|u\|_{s}^{2}=\|u\|_{L^{2}(Q)}^{2}+\|u\|_{\mathbb{H}(Q)}^{2}$. Hence, it remains to be shown that $\|u\|_{L^{2}(Q)} \lesssim\|u\|_{\mathbb{H}(Q)}$. We associate with each $u \in \mathbb{H}_{0}(Q)$ an element $v(u) \in \mathbb{H}_{T}(Q)$ through the dual problem:

$$
\left(S_{\lambda} w, v(u)\right)_{L^{2}(Q)}=(w, u)_{L^{2}(Q)} \quad \forall w \in \mathbb{H}_{0}(Q) .
$$

The dual bilinear form being coercive and bounded, it holds that $\|v(u)\|_{\mathbb{H}(Q)} \lesssim\|u\|_{\mathbb{H}(Q)}$. The boundedness of the bilinear form in turn leads to the sequence of inequalities:

$$
\|u\|_{\mathbb{H}(Q)}^{2} \gtrsim\|u\|_{\mathbb{H}(Q)}\|v(u)\|_{\mathbb{H}(Q)} \gtrsim\left(S_{\lambda} u, v(u)\right)_{L^{2}(Q)}=(u, u)_{L^{2}(Q)}
$$


which confirms the equivalence.

We restrict ourselves to a consideration of the interior regularity of solutions of (4.1). Essentially, we proceed in a similar manner as $[6, \S 7.3 .2]$. On account of its compliance with the premises of the generalized Lax-Milgram theorem, the bilinear form $\left(S_{\lambda} \cdot, \cdot\right)_{L^{2}(Q)}$ is weakly coercive on $\mathbb{H}_{0}(Q) \times \mathbb{H}_{T}(Q)$ :

$$
\inf _{u \in \mathbb{H}_{0}(Q)} \sup _{v \in \mathbb{H}_{T}(Q)} \frac{\left(S_{\lambda} u, v\right)_{L^{2}(Q)}}{\|u\|_{\mathbb{H}(Q)}\|v\|_{\mathbb{H}(Q)}} \geq \gamma>0
$$

for some positive constant $\gamma$. Let us now define the isometric operator $G: \mathbb{H}_{0}(Q) \rightarrow \mathbb{H}_{T}(Q)$ through the map

$$
u \mapsto G u=\underset{v \in \mathcal{V}}{\arg \sup } \frac{\left(S_{\lambda} u, v\right)_{L^{2}(Q)}}{\|u\|_{\mathbb{H}(Q)}\|v\|_{\mathbb{H}(Q)}},
$$

where $\mathscr{V}=\left\{v \in \mathbb{H}_{T}(Q):\|v\|_{\mathbb{H}(Q)}=\|u\|_{\mathbb{H}(Q)}\right\}$. By virtue of the weak coercivity (4.5) and the equivalence of $\|\cdot\|_{1}$ and $\|\cdot\|_{\mathbb{H}(Q)}$ on $\mathbb{H}_{0}(Q)$ it thus holds that $\left(S_{\lambda} u, G u\right)_{L^{2}(Q)} \geq \gamma\|u\|_{\mathbb{H}(Q)}^{2} \gtrsim\|u\|_{1}^{2}$ for all $u \in \mathbb{H}_{0}(Q)$. Let $q$ be a bounded open subset of the space/time cylinder $Q$ such that the closure of $q$ is contained in $Q$. We denote by $\mathbb{H}_{0}^{s}(q)$ the closure of $\mathscr{D}(q)$ in the $\|\cdot\|_{s}$-norm. Extending $u$ to a distribution on $\mathbb{R}^{2}$ and invoking the Fourier transform, we obtain

$$
\widehat{G u}^{*}(\kappa, \omega) \widehat{S u}(\kappa, \omega) \gtrsim\left(1+\kappa^{4}+\omega^{2}\right)|\hat{u}(\kappa, \omega)|^{2},
$$

where $(\cdot)^{*}$ represents complex conjugation and $\widehat{S u}$ and $\widehat{G u}$ represent the Fourier transforms of $S_{\lambda} u$ and $G u$,

$$
\widehat{S u}(\kappa, \omega)=\left(\lambda^{2} \kappa^{4}-\omega^{2}\right) \hat{u}(\kappa, \omega), \quad \widehat{G u}(\kappa, \omega)=\beta\left(\lambda^{2} \kappa^{4}-\omega^{2}\right)\left(\kappa^{4}+\omega^{2}\right)^{-1} \hat{u}(\kappa, \omega),
$$

respectively; see Appendix B. The constant $\beta$ is determined by $\|G u\|_{\mathbb{H}(Q)}=\|u\|_{\mathbb{H}(Q)}$. Equation (4.7a) leads to the following sequence of bounds:

$$
\begin{gathered}
\|u\|_{s}^{2}=\int_{\mathbb{R}^{2}}\left(1+\kappa^{4}+\omega^{2}\right)^{s}|\hat{u}(\kappa, \omega)|^{2} d \kappa d \omega \lesssim \int_{\mathbb{R}^{2}}\left(1+\kappa^{4}+\omega^{2}\right)^{s-1} \widehat{G u} \widehat{G}^{*}(\kappa, \omega) \widehat{S u}(\kappa, \omega) d \kappa d \omega \\
=\int_{\mathbb{R}^{2}}\left(1+\kappa^{4}+\omega^{2}\right)^{s / 2} \widehat{G u}^{*}(\kappa, \omega)\left(1+\kappa^{4}+\omega^{2}\right)^{(s-2) / 2} \widehat{\operatorname{Su}}(\kappa, \omega) d \kappa d \omega \\
\leq\left(\int_{\mathbb{R}^{2}}\left(1+\kappa^{4}+\omega^{2}\right)^{s}|\widehat{G u}(\kappa, \omega)|^{2} d \kappa d \omega\right)^{1 / 2}\left(\int_{\mathbb{R}^{2}}\left(1+\kappa^{4}+\omega^{2}\right)^{s-2}|\widehat{S u}(\kappa, \omega)|^{2} d \kappa d \omega\right)^{1 / 2},
\end{gathered}
$$

i.e., $\|u\|_{s}^{2} \lesssim\|G u\|_{s}\|S u\|_{s-2}$. However, equation (4.7b) moreover conveys that $\|G u\|_{s} \lesssim\|u\|_{s}$ and, accordingly, $\|u\|_{s} \lesssim\|S u\|_{s-2}$. We can now proceed in a similar manner as [6, pp.430-433] to show the following interior-regularity theorem: if $u \in \mathbb{H}_{0}(Q)$ and $S u \in \mathbb{H}_{\text {loc }}^{s}(Q)(s \geq-1)$ then $u \in \mathbb{H}_{\text {loc }}^{s+2}(Q)$, where

$$
\mathbb{H}_{\text {loc }}^{s}(Q)=\left\{u \in \mathscr{D}^{\prime}(Q): \phi u \in \mathbb{H}^{s}(Q) \text { for all } \phi \in \mathscr{D}(Q)\right\}
$$

This means that the interior regularity of the solution of (4.1) increases with the interior regularity of the right member $b$.

Let us now establish the relation between the regularity of the pre-smoothing error and the postsmoothing error. Suppose that the pre-smoothing error $e$ resides in $\mathbb{H}_{\text {loc }}^{s}(Q)(s \geq 1)$. From $(2.1 \mathrm{~d})$ and (2.17) it follows that the Fourier transform of $P_{\nu} e$ is bounded by

$$
|\widehat{P e}|^{2} \lesssim\left(1+\kappa^{2}+\omega^{2}\right)^{3 / 2}|\hat{e}(\kappa, \omega)|^{2} .
$$

Hence,

$$
\left\|P_{\nu} e\right\|_{r}^{2}=\int_{\mathbb{R}^{2}}\left(1+\kappa^{4}+\omega^{2}\right)^{r}|\widehat{P e}|^{2} d \kappa d \omega \lesssim \int_{\mathbb{R}^{2}}\left(1+\kappa^{4}+\omega^{2}\right)^{r+3 / 2}|\hat{e}|^{2} d \kappa d \omega
$$


Equation (4.11) conveys that $P_{\nu}$ e resides in $\mathbb{H}_{\text {loc }}^{s-3 / 2}(Q)$. Therefore, if $b=-\mu P_{\nu} e$ acts as a right member for (4.1), in accordance with the error amplification relation for the defect-correction process (3.11), then $e \in \mathbb{H}_{\text {loc }}^{s+1 / 2}(Q)$ and, indeed, the post-smoothing error possesses higher regularity than the presmoothing error. The results of section 3.3 can now be extended mutatis mutandis to the model problem and, in particular, the defect-correction process (3.10) provides an asymptotically-perfect smoother for hierarchical finite-element discretizations of the model problem (2.12).

\subsection{The space/time multigrid process}

For the model problem, the defect-correction process (3.10) corresponds to a standard iterative process for fluid-structure-interaction problems, viz., the subiteration (or successive approximation) process. Given an initial approximation for the displacement field of the structure, the subiteration process is defined by the following iterative process: for $i=1,2, \ldots$

(1) transfer the displacement of the structure at the fluid/structure interface to the fluid,

(2) solve the initial-boundary-value problem for the fluid subject to the prescribed displacement,

(3) transfer the pressure of the fluid at the fluid/structure interface to the structure,

(4) solve the initial-boundary-value problem for the structure subject to the prescribed pressure.

It is to be noted that in the model problem the operations (1)-(3) are condensed into the dtp operator. Hence, the multigrid process that we propose essentially corresponds to subiteration enhanced with coarse-grid correction.

An important implication of the relation $S_{\lambda}^{-1} P_{\nu}: \mathbb{H}_{\mathrm{loc}}^{s}(Q) \rightarrow \mathbb{H}_{\mathrm{loc}}^{s+1 / 2}(Q)$ is that the defectcorrection process (3.10) smoothens the error in space/time. Hence, the smoothing based on (3.10) enables us to extract the coarse-grid correction from a finite-element space that is coarser in both space and time. This motivates the predicate space/time multigrid. Of course, the coarsening can optionally be restricted to the spatial direction only, as a form of semi-coarsening.

We have restricted ourselves so far to a consideration of the two-grid method. It is to be noted, however, that the coarse-grid problem (3.6) and the fine-grid problem (3.3) are uniform. On account of the asymptotic perfection of the smoothing process provided by (3.10), on sufficiently fine coarse-grid spaces the coarse-grid problem can thus again be solved by one iteration of (3.10) and coarse-grid correction. Let us allude to the implied recursion. On coarser grids, one iteration of (3.10) potentially provides insufficient smoothing to render the error in the projection of the post-smoothing error $e \in \mathbb{H}_{\mathrm{loc}}^{s+1 / 2}(Q)$ onto the approximation space $U_{l}$ suitably small. However, by recursion, the postsmoothing error can be further regularized by means of multiple iterations of (3.10). In particular, $n$ iterations of (3.10) confine the post-smoothing error to $\mathbb{H}_{\mathrm{loc}}^{s+n / 2}(Q)$. In general, the increased regularity of the post-smoothing error provided by multiple iterations of (3.10) yields a significant reduction in the projection error, unless the polynomial degree of the approximation space $U_{l}$ is restrictive; cf. the interpolation-error estimate (3.19). On the coarsest mesh, the approximation problem can generally be solved at negligible computational expense by means of any potentially inefficient solver. If the defectcorrection process (3.10) separately converges, then (3.10) can serve for this purpose. Otherwise, the interface-GMRES method can be used, for instance; see $[5,15]$.

In multigrid algorithms the initial approximation for each but the coarsest approximation problem is typically generated by means of injection of the solution on the next coarser mesh. This procedure is referred to as full multigrid; see [3, $\S 7]$. Denoting by $u$ the actual solution to $(2.12)$ and by $u^{l-1}$ and $u^{l}$ the approximate solutions on approximation spaces $U_{l-1}$ and $U_{l}$, respectively, the error in the injected initial approximation on $\tilde{U}_{l}$ is bounded by

$$
\left\|u^{l}-u^{l-1}\right\|_{\mathbb{H}(Q)} \leq\left\|u^{l}-u\right\|_{\mathbb{H}(Q)}+\left\|u^{l-1}-u\right\|_{\mathbb{H}(Q)} .
$$

The right member of (4.12) can be identified as the sum of the discretization errors on $U_{l}$ and $U_{l-1}$. In general, it holds that $\left\|u^{l-1}-u\right\|_{\mathbb{H}(Q)} \leq C\left\|u^{l}-u\right\|_{\mathbb{H}(Q)}$ for some bounded constant $C$ depending on the 
mesh sizes and polynomial orders of approximation pertaining to $U_{l}$ and $U_{l-1}$. Hence, the error in the initial approximation obtained by injection is within a constant factor of the discretization error. Denoting by $\tilde{u}^{l}$ the approximation obtained by one iteration of the two-grid method, it holds that

$$
\left\|\tilde{u}^{l}-u^{l}\right\|_{\mathbb{H}(Q)} /\left\|u^{l}-u\right\|_{\mathbb{H}(Q)} \leq C\left\|\tilde{u}^{l}-u^{l}\right\|_{\mathbb{H}(Q)} /\left\|u^{l-1}-u^{l}\right\|_{\mathbb{H}(Q)}
$$

However, by virtue of the asymptotic perfection of the smoothing process, the right member tends to 0 as $l \rightarrow \infty$, i.e., on sufficiently fine approximation spaces one iteration of the two-grid method renders the evaluation error $\left\|\tilde{u}^{l}-u^{l}\right\|_{\mathbb{H}(Q)}$ negligible compared to the discretization error $\left\|u^{l}-u\right\|_{\mathbb{H}(Q)}$. Since further reduction of the evaluation error does not improve the approximation to the continuum solution $u$ anyway, one iteration of the two-grid method suffices. It is to be noted that if an arbitrary initial approximation is used, then multiple two-grid iterations will generally be required to reduce the evaluation error below the discretization error, as the discretization error typically vanishes faster as the approximation space is refined than the convergence of the two-grid method improves.

\section{Numerical experiments}

To test the space/time multigrid method for the model problem, we conduct numerical experiments at various representative settings of the parameters. In section 5.1 we present the setup of the discretization of the model problem. Section 5.2 investigates the properties of the defect-correction process separately. In particular, this section serves to elucidate the nonnormality of the defect-correction process for the panel-model problem. Section 5.3 is devoted to the asymptotically-perfect-smoothing property of the defect-correction process. Finally, in section 5.4 we consider a variant of the smoothing process based on local defect-correction within each time step, corresponding to a so-called looselycoupled partitioned method (see, e.g., [14]) with coarse-grid correction. This investigation is motivated by the predominance of loosely-coupled partitioned methods in practical fluid-structure-interaction computations.

\subsection{Setup of the discrete approximation problems}

Let $Q$ represent a partition of the space time cylinder $Q$ into $N=N_{x} \times N_{t}$ uniform quadrilateral elements, i.e., each element $Q_{e}$ has length $h=N_{x}^{-1}$ and $\tau=T N_{t}^{-1}$ in space and time, respectively. We consider finite-element approximations based on the piecewise-polynomial space:

$$
\mathscr{P}(\boldsymbol{Q}, d)=\left\{u \in L^{2}(Q):\left.u\right|_{Q_{e}} \text { is polynomial of degree } d\right\} .
$$

for some natural number $d$. Because the approximation space $\mathscr{P}(\boldsymbol{Q}, d)$ admits interelement discontinuities, it is nonconforming, i.e., $\mathscr{P}(\boldsymbol{Q}, d) \not \subset \mathbb{H}(Q)$. We accommodate this nonconformity by means of Lagrange multipliers on the spatial interelement edges and weakly-enforced initial conditions on the temporal interelement edges. The latter implies that the discretization is time discontinuous. The Lagrange-multiplier formalism is moreover used to enforce the boundary conditions at the lateral boundaries.

The set of edges corresponding to $Q$ is denoted by $\Gamma$ :

$$
\boldsymbol{\Gamma}=\left\{\gamma_{e} \subset \text { closure } Q: \gamma_{e}=\operatorname{int}\left(\operatorname{closure} Q_{e} \cap \text { closure } \bar{Q}_{e}\right), \forall\left(Q_{e}, \bar{Q}_{e}\right) \in \boldsymbol{Q} \times \boldsymbol{Q}, Q_{e} \neq \bar{Q}_{e}\right\} .
$$

We separate $\boldsymbol{\Gamma}$ into 6 complementary sets, viz., the left, right and interior spatial edges,

$$
\begin{array}{ll}
\boldsymbol{S}_{0}=\left\{\gamma_{e} \in \boldsymbol{\Gamma}: \gamma_{e} \subset\{0\} \times\right] 0, T[\}, & \boldsymbol{S}_{1}=\left\{\gamma_{e} \in \boldsymbol{\Gamma}: \gamma_{e} \subset\{1\} \times\right] 0, T[\}, \\
& \boldsymbol{S}_{\mathrm{int}}=\left\{\gamma_{e} \in \boldsymbol{\Gamma}: \gamma_{e} \subset\left\{h, 2 h, \ldots,\left(N_{x}-1\right) h\right\} \times\right] 0, T[\},
\end{array}
$$

and the bottom, top and interior temporal edges,

$$
\begin{aligned}
\boldsymbol{T}_{0}=\left\{\gamma_{e} \in \boldsymbol{\Gamma}: \gamma_{e} \subset \Omega \times\{0\}\right\}, \quad \boldsymbol{T}_{T}= & \left\{\gamma_{e} \in \boldsymbol{\Gamma}: \gamma_{e} \subset \Omega \times\{T\}\right\}, \\
& \boldsymbol{T}_{\text {int }}=\left\{\gamma_{e} \in \boldsymbol{\Gamma}: \gamma_{e} \subset \Omega \times\left\{\tau, 2 \tau, \ldots,\left(N_{t}-1\right) \tau\right\}\right\} .
\end{aligned}
$$


To accommodate the Lagrange multipliers, we associate to the spatial-edge set $\boldsymbol{S}=\boldsymbol{S}_{0} \cup \boldsymbol{S}_{1} \cup \boldsymbol{S}_{\text {int }}$ the edge-function space:

$$
\mathscr{E}(\boldsymbol{S}, d)=\left\{u \in L^{2}(\boldsymbol{S}):\left.u\right|_{\gamma_{e}} \text { is polynomial of degree } d \text { for all } \gamma_{e} \in \boldsymbol{S}\right\}
$$

To condense the notation, we define $(\cdot)^{ \pm}=\lim _{\epsilon \rightarrow+0}(\cdot) \pm \epsilon$. For instance, for $(x, t) \in \gamma_{e} \in S_{\text {int }}$ it holds that $\left(x^{+}, t\right)$ and $\left(x^{-}, t\right)$ are just right and left of $\gamma_{e}$, respectively. Moreover, we introduce the trilinear form $z: \mathscr{P}(\boldsymbol{Q}, d) \times \mathscr{E}(\boldsymbol{S}, d) \times \mathscr{E}(\boldsymbol{S}, d) \rightarrow \mathbb{R}:$

$$
\begin{aligned}
z\left(u, h_{0}, h_{1}\right)=\sum_{\gamma_{e} \in \boldsymbol{S}_{\mathrm{int}}}\left(\left(h_{0}, u\left(x^{+}, \cdot\right)\right.\right. & \left.\left.-u\left(x^{-}, \cdot\right)\right)_{L^{2}\left(\gamma_{e}\right)}+\left(h_{1}, D u\left(x^{+}, \cdot\right)-D u\left(x^{-}, \cdot\right)\right)_{L^{2}\left(\gamma_{e}\right)}\right) \\
& +\sum_{\gamma_{e} \in \boldsymbol{S}_{0} \cup \boldsymbol{S}_{1}}\left(\left(h_{0}, u\left(x^{ \pm}, \cdot\right)\right)_{L^{2}\left(\gamma_{e}\right)}+\left(h_{1}, D u\left(x^{ \pm}, \cdot\right)\right)_{L^{2}\left(\gamma_{e}\right)}\right),
\end{aligned}
$$

where the sign in the second sum is chosen such that $\left(x^{ \pm}, t\right)$ lies in $Q$. The approximation problem based on Lagrange multipliers and weakly-enforced initial conditions can now be condensed into: find $\left(u, g_{0}, g_{1}\right) \in \mathscr{P}(\boldsymbol{Q}, d) \times \mathscr{E}(\boldsymbol{S}, d) \times \mathscr{E}(\boldsymbol{S}, d)$ such that

$$
\bar{a}_{\mu}\left(\left(u, g_{0}, g_{1}\right),\left(v, h_{0}, h_{1}\right)\right)=\bar{b}_{\mu}\left(\left(v, h_{0}, h_{1}\right)\right) \quad \forall\left(v, h_{0}, h_{1}\right) \in \mathscr{P}(\boldsymbol{Q}, d) \times \mathscr{E}(\boldsymbol{S}, d) \times \mathscr{E}(\boldsymbol{S}, d),
$$

where the corresponding bilinear and linear forms are defined as:

$$
\begin{gathered}
\bar{a}_{\mu}\left(\left(u, g_{0}, g_{1}\right),\left(v, h_{0}, h_{1}\right)\right)=z\left(u, h_{0}, h_{1}\right)+z\left(v, g_{0}, g_{1}\right)+\sum_{Q_{e} \in \boldsymbol{Q}}\left((S u, v)_{L^{2}\left(Q_{e}\right)}+\mu\left(P_{\nu} u, v\right)_{L^{2}\left(Q_{e}\right)}\right) \\
+\sum_{\gamma_{e} \in \boldsymbol{T}_{\mathrm{int}}}\left(\left(u^{\prime}\left(\cdot, t^{+}\right)-u^{\prime}\left(\cdot, t^{-}\right), v\left(\cdot, t^{+}\right)\right)_{L^{2}\left(\gamma_{e}\right)}-\left(u\left(\cdot, t^{+}\right)-u\left(\cdot, t^{-}\right), v^{\prime}\left(\cdot, t^{+}\right)\right)_{L^{2}\left(\gamma_{e}\right)}\right) \\
+\sum_{\gamma_{e} \in \boldsymbol{T}_{0}}\left(\left(u^{\prime}\left(\cdot, t^{+}\right), v\left(\cdot, t^{+}\right)\right)_{L^{2}\left(\gamma_{e}\right)}-\left(u\left(\cdot, t^{+}\right), v^{\prime}\left(\cdot, t^{+}\right)\right)_{L^{2}\left(\gamma_{e}\right)}\right) \\
\bar{b}_{\mu}\left(\left(v, h_{0}, h_{1}\right)\right) \\
=\sum_{\gamma_{e} \in \boldsymbol{T}_{0}}\left(\left(\varphi_{1}, v\left(\cdot, t^{+}\right)\right)_{L^{2}\left(\gamma_{e}\right)}-\left(\varphi_{0}, v^{\prime}\left(\cdot, t^{+}\right)\right)_{L^{2}\left(\gamma_{e}\right)}\right) .
\end{gathered}
$$

Upon introducing suitable bases $\left\{\vartheta_{i}\right\}$ and $\left\{\zeta_{i}\right\}$ for $\mathscr{P}(\boldsymbol{Q}, d)$ and $\mathscr{E}(\boldsymbol{S}, d)$, equation (5.6) translates into a system of linear algebraic equations $A_{i j} q_{j}=b_{i}$, where

$$
A_{i j}=\bar{a}_{\mu}\left(\left(\vartheta_{k(j)}, \zeta_{l(j)}, \zeta_{m(j)}\right),\left(\vartheta_{k(i)}, \zeta_{l(i)}, \zeta_{m(i)}\right)\right), \quad b_{i}=\bar{b}\left(\left(\vartheta_{k(i)}, \zeta_{l(i)}, \zeta_{m(i)}\right)\right),
$$

and $i \mapsto(k, l, m)$ represents a renumbering scheme, i.e., an isomorphism between the index $i$ and the multi-index $(k, l, m)$. The approximate solution $u \in \mathscr{P}(\boldsymbol{Q}, d)$ can be composed as $u=\sum_{k} q_{i(k)} \vartheta_{k}$.

\subsection{Nonnormality of the defect-correction process}

The defect-correction process (3.10) is one of the principal elements of the multigrid process. To elucidate the properties of the defect-correction process for the model problem, we first consider the convergence behavior of (3.10) separately. We select the initial conditions in accordance with the displacement the first eigenmode of the beam:

$$
\varphi_{0}(x)=c_{1} \sin (a x)+c_{2} \cos (a x)+c_{3} \sinh (a x)+c_{4} \cosh (a x), \quad \varphi_{1}(x)=0,
$$

with $a \approx 4.730$ the smallest root of $\cos (a) \cosh (a)-1$, such that $\varphi_{0}(x)$ admits nontrivial $c_{i}$ subject to the boundary conditions $\left.\varphi_{0}\right|_{\partial \Omega}=\left.D \varphi_{0}\right|_{\partial \Omega}=0$. The constants $c_{i}$ are scaled such that $c_{1}^{2}+\cdots+c_{4}^{2}=1$. Next, we generate an initial approximation $\tilde{u}_{0}$ by solving the structure problem separately, so that the initial approximation complies with the initial and boundary conditions. 

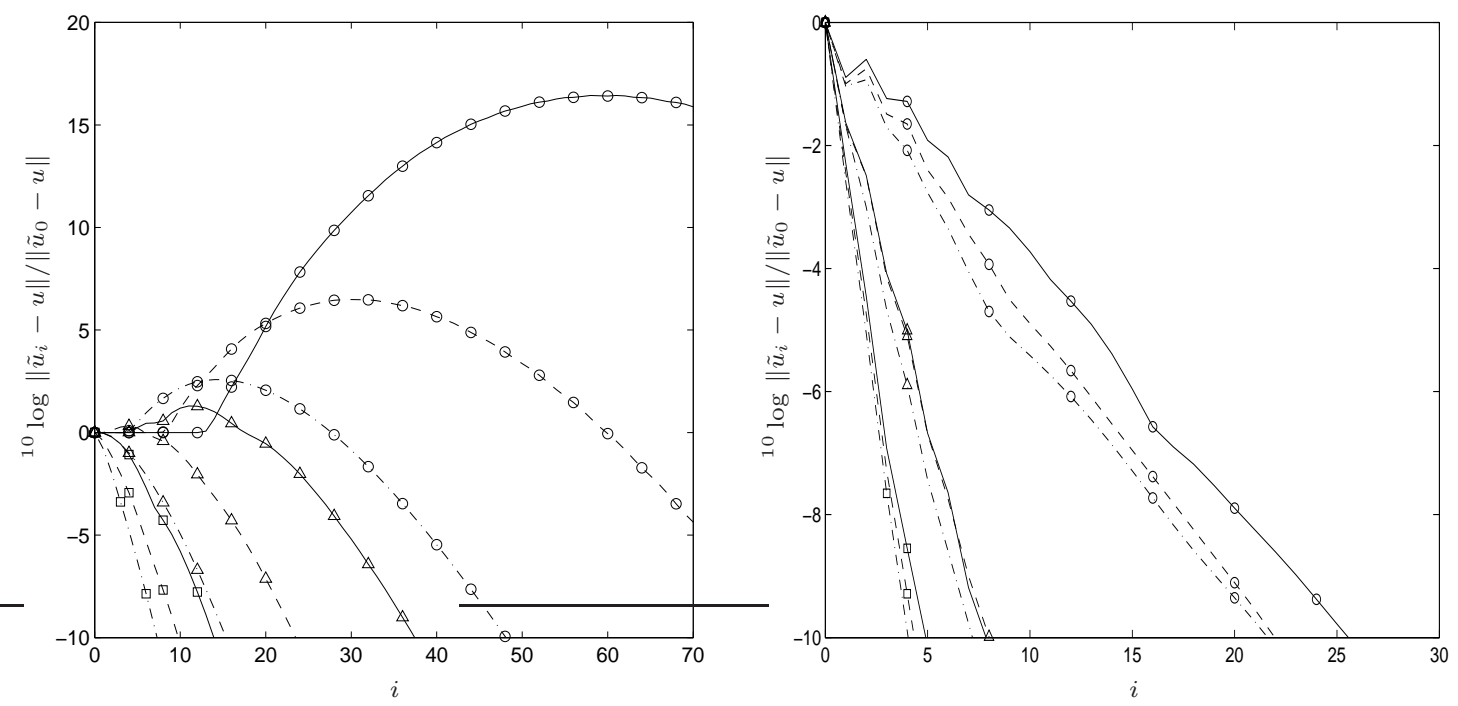

Figure 2: Error reduction $\left\|\tilde{u}_{i}-u\right\|_{\mathbb{H}(Q)} /\left\|\tilde{u}_{0}-u\right\|_{\mathbb{H}(Q)}$ versus the iteration counter for the defect correction process separately (left) and the two-grid method (right) for $T=1(-\cdot), T=2(--)$, $T=4(-)$ and $\mu=10^{-1}(\square), \mu=10^{0}(\triangle)$ and $\mu=10^{1}(\circ)$.

Figure 2 (left) plots the error reduction $\left\|\tilde{u}_{i}-u\right\|_{\mathbb{H}(Q)} /\left\|\tilde{u}_{0}-u\right\|_{\mathbb{H}(Q)}$ for $\lambda=10^{-2}, \nu=1.5$ and, $T=1,2,4$ and $\mu=10^{-1}, 10^{0}, 10^{1}$. The finite-element space is characterized by mesh-size $(h, \tau)=$ $(64,32)$ and polynomial degree $d=3$. Note that the number of elements in temporal direction per unit time is 32 , independent of $T$. The figure exhibits that for large $\mu$ and $T$, the error can increase before convergence sets in. This behavior is induced by the nonnormality of the subiteration process, i.e., the error-amplification operator $E=\mu S_{\lambda}^{-1} P_{\nu}$ is nonnormal; see also [4,9,17]. As a result of the nonnormality, the norm $\|E\|$ can be much larger than the spectral radius $\operatorname{spr}(E)$. Moreover, for nonnormal operators the condition-number of the projection onto the eigenvectors of $E$, here denoted by $\varkappa(E)$, can be very large. In view of the bounds

$$
\left\|E^{n}\right\| \leq\|E\|^{n}, \quad\left\|E^{n}\right\| \leq \varkappa(E)(\operatorname{spr}(E))^{n},
$$

if $\operatorname{spr}(E)<1<\|E\|$ then the error can increase before the asymptotic convergence indicated by the spectral radius sets in. For instance, for $T=4$ and $\mu=10$ the error increases by a factor $10^{16}$ before it convergences. As $\mu$ and $T$ decrease, the norm $\|E\|$ decreases and, hence, for sufficiently small $\mu$ and $T,\|E\|<1$ and the convergence behavior is monotonous.

Figure 2 (right) plots the sequence of error reductions generated by the two-grid method with one iteration of (3.10) followed by a coarse-grid correction from a mesh with $(h, \tau)=(32,16)$. The figure shows that the two-grid method displays essentially monotonous convergence. Moreover, the convergence behavior of the two-grid method is essentially $T$-independent, in contrast to the strong $T$-dependence of the underlying defect-correction method. This can be assigned to the fact that the $T$-dependent behavior of the defect-correction process is induced by smooth error components, which are in the two-grid method effectively eliminated by the coarse-grid correction.

\subsection{Asymptotic perfection of the smoothing process}

To illustrate the asymptotic perfection of the smoothing provided by the defect-correction process according to (3.10), Figure 3 plots the error in the sequence of approximations $\left\{\tilde{u}_{i}\right\}$ generated by the two-grid method with one iteration of (3.10) versus the iteration counter on meshes with $d=3$ and $(h, \tau)=2^{-l}\left(2^{-2}, 2^{-1}\right), l=\{1,2,3,4\}$, for a supersonic setting $(\nu=1.5$, left $)$ and a subsonic 

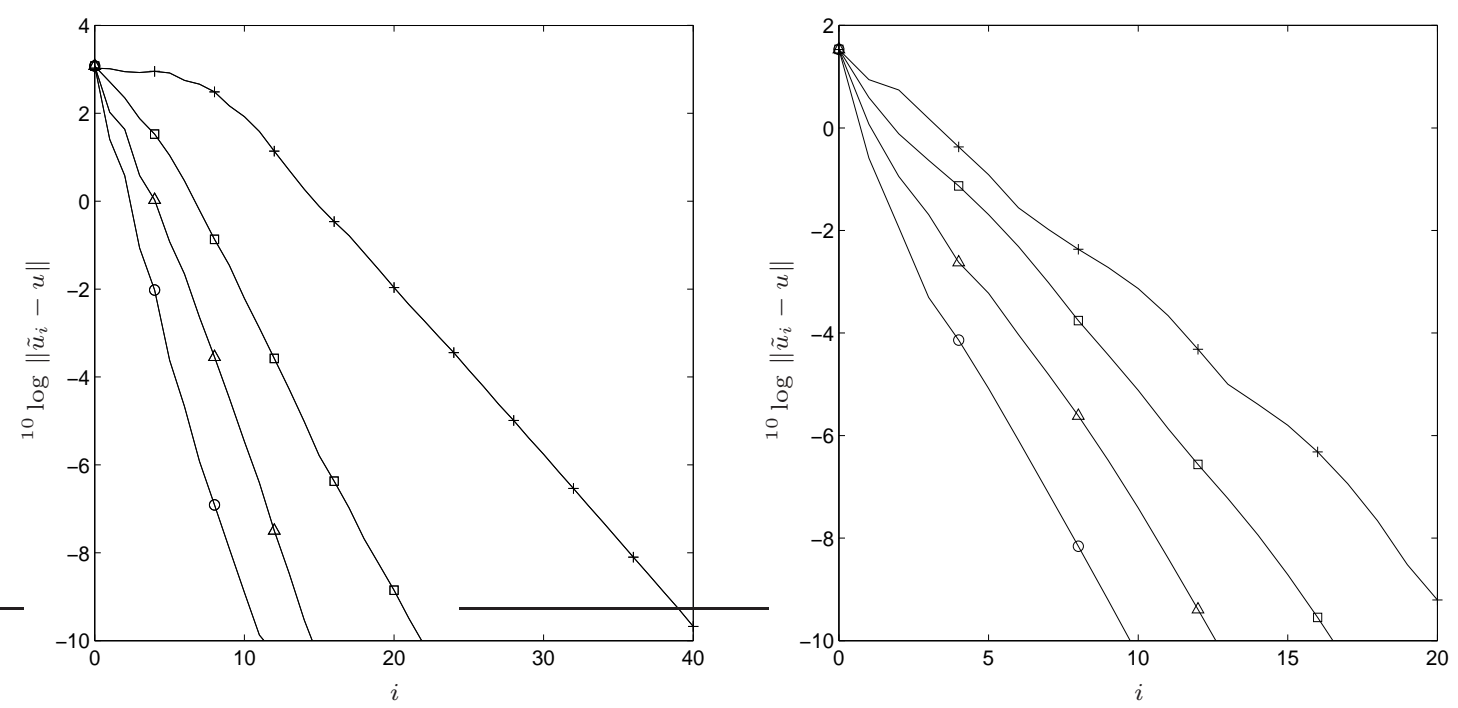

Figure 3: Error $\left\|\tilde{u}_{i}-u\right\|_{\mathbb{H}(Q)}$ versus the iteration counter for the sequence of approximations $\left\{\tilde{u}_{i}\right\}$ generated by the two-grid method for $\nu=1.5$ (left) and $\nu=0.5$ (right), and for $(h, \tau)=2^{-l}\left(2^{-2}, 2^{-1}\right), l=1(+), l=2(\square), l=3(\triangle)$ and $l=4(\circ)$.

setting $\left(\nu=0.5\right.$, right). The auxiliary parameters are set to $T=2, \lambda=10^{-2}$ and $\mu=1$. The figure corroborates that the convergence of the two-grid method improves as the mesh is refined, in accordance with the asymptotically-perfect-smoothing property of (3.10) for the model problem. The figure confirms that this behavior is independent of the Mach number $\nu$, although the details of the convergence behavior do depend on $\nu$.

To elucidate the relation between the discretization error and the evaluation error in the fullmultigrid procedure, Table 1 lists the error in the initial approximation obtained by injection of the coarse grid, $\left\|u^{l-1}-u^{l}\right\|_{\mathbb{H}(Q)}$, and the error after one iteration of the two-grid method with one iteration of the defect-correction process, $\left\|\tilde{u}^{l}-u^{l}\right\|_{\mathbb{H}(Q)}$, for $(h, \tau)=2^{-l}\left(2^{-3}, 2^{-2}\right)$ for $l=1,2,3,4$. The auxiliary parameters are identical to the subsonic case above. The supersonic test case yields similar results (results not displayed). The rows (columns) in the table indicated by ratio represent the ratios of the entries in the contiguous rows (columns). The error in the injected coarse-grid solution provides an accurate estimate of the actual coarse-grid discretization error. The entries in the first row of Table 1 therefore indicate that $\left\|u^{l}-u\right\|_{\mathbb{H}(Q)} \propto h^{2}+\tau^{2}$. This result is in accordance with finite-elementapproximation theory for the considered polynomial degree $d=3$ and norm $\|\cdot\|_{\mathbb{H}(Q)}$. In Table 1 , the asymptotically-perfect-smoothing property of the defect-correction process manifest itself through the fact that the ratio $\left\|\tilde{u}^{l}-u^{l}\right\|_{\mathbb{H}(Q)} /\left\|u^{l-1}-u^{l}\right\|_{\mathbb{H}(Q)}$ decreases as the mesh is refined. On coarse meshes, the effectiveness of the two-grid method is insufficient to reduce the evaluation error below the discretization error by a single iteration. For instance, for $l=1$ one iteration of the two-grid method yields the norm of the evaluation error $1.49510^{0}$, whereas the norm of the discretization error is approximately $8.14710^{-1}$. On account of the asymptotic perfection of the smoothing process, however, on finer meshes one iteration of the two-grid method renders the evaluation error smaller than the discretization error. Indeed, for $l=2$ the evaluation error after one iteration is $1.82210^{-1}$, whereas the discretization error is approximately $2.02910^{-1}$. For $l=3$, the subordinance of the evaluation error after one iteration $\left(2.03110^{-2}\right)$ to the discretization error (appr. $\left.5.0811^{-2}\right)$ is even more pronounced, in accordance with the theory of section 4.2.

The ratio-ratio entries in Table 1 indicate that the convergence rate of the two-grid method is approximately proportional to $|(h, \tau)|=\sqrt{h^{2}+\tau^{2}}$ : if the mesh width is halved, than the conver- 


\begin{tabular}{|c|c|c|c|c|c|c|c|}
\cline { 2 - 8 } \multicolumn{1}{c|}{} & $l=1$ & ratio & $l=2$ & ratio & $l=3$ & ratio & $l=4$ \\
\hline$\left\|u^{l-1}-u^{l}\right\|_{\mathbb{H}(Q)}$ & $3.53010^{0}$ & $2.30810^{-1}$ & $8.14710^{-1}$ & $2.49010^{-1}$ & $2.02910^{-1}$ & $2.50410^{-1}$ & $5.08110^{-2}$ \\
\hline ratio & $4.23510^{-1}$ & $5.28210^{-1}$ & $2.23710^{-1}$ & $4.47710^{-1}$ & $1.00110^{-1}$ & $5.17510^{-1}$ & $5.18210^{-2}$ \\
\hline$\left\|\tilde{u}^{l}-u^{l}\right\|_{\mathbb{H}(Q)}$ & $1.49510^{0}$ & $1.21910^{-1}$ & $1.82210^{-1}$ & $1.11510^{-1}$ & $2.03110^{-2}$ & $1.29610^{-1}$ & $2.63310^{-3}$ \\
\hline
\end{tabular}

Table 1: Convergence results for the full-multigrid method: error in the initial approximation obtained by injection of the coarse-grid solution, $\left\|u^{l-1}-u^{l}\right\|_{\mathbb{H}(Q)}$, and error after one iteration of the two-grid method, $\left\|\tilde{u}^{l}-u^{l}\right\|_{\mathbb{H}(Q)}$.

gence rate improves by approximately a factor 2 . This mesh-width dependence of the convergence rate surpasses the expectation created by the theory in sections 3.3 and 4.1, which indicates that the convergence rate is proportional to $|(h, \tau)|^{1 / 2}$. Conjecturally, the discrepancy in the predicted and observed convergence behavior can be assigned to the anisotropy of the norm $\|\cdot\|_{\mathbb{H}(Q)}$ in space/time, which depends on the second-order spatial derivative, $D^{2}(\cdot)$, and the first-order temporal derivative, $(\cdot)^{\prime}$. The theoretical convergence rate has been derived from interpolation-error estimates in (isentropic) Sobolev spaces and the inclusion relation $\mathbb{H}^{s}(Q) \subset H^{s}(Q)$ and, hence, it is not sharp. On the other hand, the numerical results in Table 1 do not unambiguously convey the asymptotic behavior of the convergence rate.

\subsection{Coarse-grid correction of loosely-coupled partitioned methods}

The defect-correction procedure (3.10) associated with subiteration represents the standard in solving fluid-structure-interaction problems. If the iteration is repeated until convergence within each time step, then one refers to the corresponding method as a strongly-coupled partitioned method. Alternatively, only a single iteration per time step can be performed. Such loosely-coupled partitioned methods are predominantly used in practical fluid-structure-interaction computations.

In sections 5.2 and 5.3 we considered global application of the defect-correction process, i.e., the defect-correction process was applied to the entire space/time cylinder $Q$, irrespective of the fact that in the discrete approximation $Q$ is composed of multiple space/time slabs $\Omega \times] t, t+\tau[$. In this section we consider local application of the defect-correction process, i.e., the defect-correction process is sequentially applied to each space/time slab in $Q$. Only a single iteration of the defect-correction process is performed per space/time slab, conforming to the loosely-coupled partitioned approach. The initial estimate on each space/time slab is obtained by solving the structure problem separately. In addition, we consider the loosely-coupled partitioned method with coarse-grid correction. The local defect-correction process then functions as a smoother in the space/time multigrid method.

Figure 4 plots the local error in each space/time slab, $\|\tilde{u}-u\|_{\mathbb{H}(\Omega \times] t_{i}-\tau / 2, t_{i}+\tau / 2[)}$, where $u$ represents the solution of the approximation problem and $\tilde{u}$ represents the approximation obtained by the looselycoupled method or by the loosely-coupled method with coarse-grid correction, versus the center of the time interval, $t_{i}=(i-1 / 2) \tau$, for $\nu=0.5$ (left) and $\nu=1.5$ (right). The auxiliary parameters are $\lambda=10^{-2}, \mu=1$ and $T=2$. Moreover, we consider two meshes, viz., a coarse mesh with $(h, \tau)=\left(2^{-5}, 2^{-4}\right)$ and a fine mesh with $(h, \tau)=\left(2^{-6}, 2^{-5}\right)$. The results in Figure 4 demonstrate that for the loosely-coupled method separately the local error grows rapidly in time. This error growth is more pronounced for large time steps: the local defect-correction process is then less effective and, accordingly, the error accumulation per time step is larger. The figure clearly illustrates that the coarse-grid correction very effectively reduces the error. It is to be noted that here the coarse-grid correction is in fact applied globally, i.e., it is applied to the entire space/time cylinder at once. Of course, local coarse-grid correction, i.e., coarse-grid correction after $2^{n}$ time steps with $n$ a small integer number, will be even more effective. The number $n$ then restricts the number of coarsening steps in the multigrid algorithm. However, this can be straightforwardly circumvented by means of semi-coarsening strategies. 

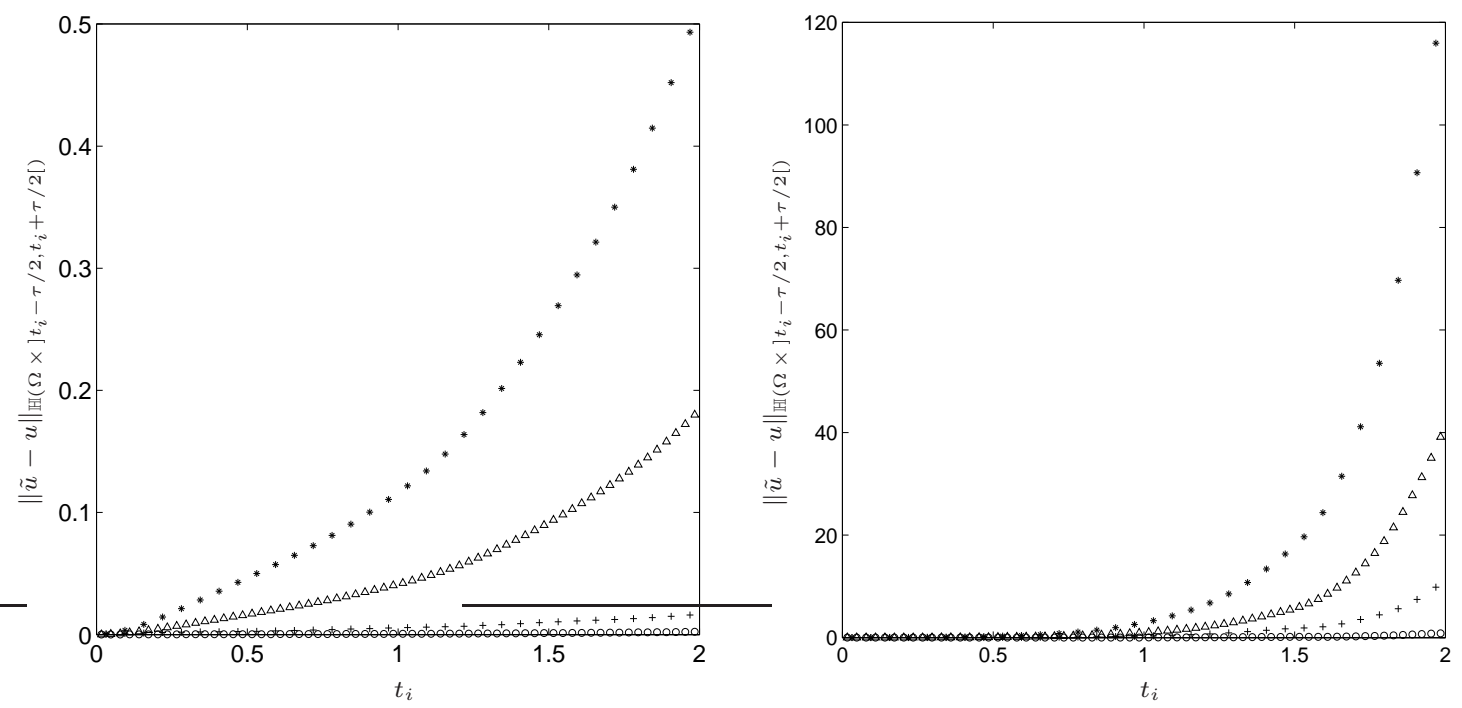

Figure 4: Local error $\|\tilde{u}-u\|_{\mathbb{H}(\Omega \times] t_{i}-\tau / 2, t_{i}+\tau / 2[)}$ versus the time-interval center $t_{i}=(i-1 / 2) \tau$ for $\nu=0.5$ (left) and $\nu=1.5$ (right): the loosely-coupled partitioned method on the coarse mesh $(*)$ and the fine mesh $(\triangle)$ and the loosely-coupled partitioned method with coarse-grid correction on the coarse mesh $(+)$ and the fine mesh $(\circ)$.

\section{Conclusion}

We developed a space/time multigrid method for an integro-differential model of a prototypical fluidstructure-interaction problem, viz., the panel problem. Based on a variational space/time formulation of the structure problem separately, we demonstrated that the displacement-to-pressure operator that represents the action of the fluid on the structure is relatively compact with respect to the structural operator. We then established a variational theory of the multigrid methodology, and we showed for a canonical variational problem that if the underlying operator admits a partitioning into a regular and a relatively-compact part, then the defect-correction process based on this partitioning potentially yields an asymptotically-perfect smoother for multigrid methods, i.e., a smoother that is perfect on sufficiently fine meshes. Supported by an interior regularity theorem for the inverse of the structure operator, we showed that the defect-correction process indeed yields an asymptoticallyperfect smoother for the fluid-structure-interaction problem. Hence, on sufficiently fine approximation spaces the fluid-structure-interaction problem can be solved to arbitrary accuracy by one iteration of the defect-correction process followed by a coarse-grid correction. We alluded to the fact that the proposed multigrid method essentially consists of the standard subiteration method for fluid-structureinteraction problems enhanced with coarse-grid correction. Moreover, we noted that the coarsening in the multigrid method can be applied in space/time.

By means of numerical experiments we demonstrated that the subiteration defect-correction process displays nonnormal convergence behavior for the model problem. In particular, despite formal stability, for certain parameter settings the error in the approximation increased by many orders of magnitude before asymptotic set in. In contrast, the multigrid method displayed essentially monotonous convergence. Moreover, the numerical results confirmed the asymptotically-perfect-smoothing property of the defect-correction process, through the improvement in the convergence rate of the multigrid method as the mesh is refined. Finally, we considered numerical experiments pertaining to coarse-grid correction of loosely-coupled partitioned methods, in view of the predominance of such methods in practical fluid-structure-interaction computations. The results indicated that the coarse-grid correction very effectively reduces the error induced by the loose coupling. 
We conjecture that the relative compactness of the fluid part of the operator with respect to the structural part is a characteristic shared by many fluid-structure-interaction problems and that, accordingly, the proposed space/time multigrid method extends with the necessary modifications to other fluid-structure-interaction problems.

\section{A. An upper bound for the Fourier symbol of the integral operator $\Xi$}

Our objective is to prove the upper bound (2.17) for the modulus of the Fourier symbol $\hat{\Xi}(\cdot)$ according to (2.16). To facilitate the presentation, we introduce the transformation to polar coordinates:

$$
(\rho, \zeta) \mapsto(\kappa, \omega)=\rho(\cos \zeta, \sin \zeta) .
$$

Introducing the transformation into (2.16), we obtain:

$$
\hat{\Xi}(\rho, \zeta, x, t)=i \int_{-\pi / 2}^{\pi / 2} \chi(\rho, \zeta, t, \theta) d \theta,
$$

with

$$
\chi(\rho, \zeta, t, \theta)=\frac{1-\mathrm{e}^{-i \rho \ell(\zeta, \theta) t}}{\rho \ell(\zeta, \theta)}, \quad \ell(\zeta, \theta)=\nu \cos \zeta+\sin \zeta-\cos \zeta \sin \theta .
$$

It is to be noted that in the limit as $\rho \rightarrow+0$, it holds that $\chi(\rho, \zeta, t) \sim i t$, so that $|\hat{\Xi}| \leq C t$ near $\rho=0$ for some positive constant $C$ independent of $\rho, \zeta, t$. Our primary concern, however, is the limit $\rho \rightarrow \infty$. Multiplying $\chi$ by a factorization of unity, we obtain

$$
\hat{\Xi}(\rho, \zeta, x, t)=\int_{-\pi / 2}^{\pi / 2} \bar{\chi}(\rho, \zeta, t, \theta) \mathrm{e}^{-i \rho \ell(\zeta, \theta) t / 2} d \theta
$$

where

$$
\bar{\chi}(\rho, \zeta, t, \theta)=-\frac{2 \sin (\rho \ell(\zeta, \theta) t / 2)}{\rho \ell(\zeta, \theta)} .
$$

The function $\bar{\chi}(\cdot)$ being analytic in $\theta$, the integral (A.4) can be evaluated by means of the method of stationary phase:

$$
\hat{\Xi}(\rho, \zeta, x, t) \sim \sum_{\theta \in \Theta} \bar{\chi}(\rho, \zeta, t, \theta) \sqrt{\frac{4 \pi}{\left|\rho t \ell^{\prime \prime}(\zeta, \theta)\right|}} \mathrm{e}^{-i(\rho \ell(\zeta, \theta) t / 2+s)} \quad \text { as }|\rho| \rightarrow \infty,
$$

where $\Theta=\{-\pi / 2, \pi / 2\}$ is the set of stationary points of $\ell(\zeta, \theta), \ell^{\prime \prime}=\partial^{2} \ell / \partial \theta^{2}$ and $s$ is a multiple of $\pi / 4$ depending on the sign of $\rho \ell^{\prime \prime}(\zeta, \theta)$; see, for instance, [18, $\left.\S 11.3\right]$. Moreover, it is to be noted that if $\ell(\zeta, \theta)$ is bounded away from zero then $|\bar{\chi}| \propto|\rho|^{-1}$ as $|\rho| \rightarrow \infty$. On the other hand, it holds that $|\bar{\chi}| \sim t$ as $\ell \rightarrow 0$. Summarizing, we therefore find

$$
|\hat{\Xi}(\rho, \zeta, x, t)| \propto \begin{cases}C_{0}(t)|\rho|^{-1 / 2} & \text { if } \exists \theta \in \Theta: \ell(\zeta, \theta)=0, \\ C_{1}(t)|\rho|^{-3 / 2} & \text { otherwise, }\end{cases}
$$

as $|\rho| \rightarrow \infty$. Specifically, $C_{0}(t)$ increases as $\sqrt{t}$ and $C_{1}(t)$ decreases as $1 / \sqrt{t}$. The upper bound $(2.17)$ now follows immediately from $\rho^{2}=\kappa^{2}+\omega^{2}$.

\section{B. The Fourier symbol of $G$}

To facilitate the presentation, we use the condensed notation $(\cdot, \cdot)=(\cdot, \cdot)_{L^{2}(Q)}$ and $[\cdot, \cdot]=(\cdot, \cdot)_{\mathbb{H}(Q)}$. To determine the Fourier transform of $G u$ according to (4.6), we note that for each $u \in \mathbb{H}_{0}(Q)$ the functional $f: \mathbb{H}_{T}(Q) \rightarrow \mathbb{R}$ defined by

$$
f(v)=\frac{\left(S_{\lambda} u, v\right)}{[u, u]^{1 / 2}[v, v]^{1 / 2}},
$$


represents a bounded functional on $\mathbb{H}_{T}(Q)$. For all $v, w \in \mathbb{H}_{T}(Q)$ the functional $f$ can be expanded as $f(v+\epsilon w)=f(v)+\epsilon f^{\prime}(v, w)+\epsilon^{2} f^{\prime \prime}(v, w)+o\left(\epsilon^{2}\right)$ as $\epsilon \rightarrow 0$ with, in particular,

$$
\begin{aligned}
f^{\prime}(v, w) & =\frac{1}{[u, u]^{1 / 2}}\left(\frac{(S u, w)}{[v, v]^{1 / 2}}-\frac{(S u, v)[v, w]}{[v, v]^{3 / 2}}\right) \\
f^{\prime \prime}(v, w) & =\frac{1}{[u, u]^{1 / 2}}\left(\frac{3(S u, v)[v, w]^{2}}{2[v, v]^{5 / 2}}-\frac{(S u, v)[w, w]}{2[v, v]^{3 / 2}}-\frac{(S u, w)[v, w]}{[v, v]^{3 / 2}}\right) .
\end{aligned}
$$

The supremum of $f$ over $\mathbb{H}_{T}(Q)$ complies with the Kuhn-Tucker condition $f^{\prime}(v, w)=0$ for all $w \in$ $\mathbb{H}_{T}(Q)$. The functional $(S u, \cdot)$ being a bounded linear functional on $\mathbb{H}_{T}(Q)$, Riesz' representation theorem asserts that there exists a unique $v_{u} \in \mathbb{H}_{T}(Q)$ such that $(S u, \cdot)=\left[v_{u}, \cdot\right]$. Hence, the KuhnTucker condition can be cast into the form

$$
\left[v_{u}, w\right][v, v]=\left[v_{u}, v\right][v, w] \quad \forall w \in \mathbb{H}_{T}(Q) .
$$

One easily verifies that $v=\beta v_{u}$ satisfies (B.4) for any constant $\beta$. Moreover, the Cauchy-Schwarz inequality implies that $\left[v_{u}, w\right][w, w]^{-1 / 2} \leq\left[v_{u}, v\right][v, v]^{-1 / 2}$ for all $w \in \mathbb{H}_{T}(Q)$. This holds if and only if $v=\beta v_{u}$ and, hence, $v$ is unique modulo multiplication by a constant. Moreover, with $(S u, \cdot)=[v, \cdot]$ the Hessian $f^{\prime \prime}$ reduces to

$$
f^{\prime \prime}(v, w)=-\frac{[v, v]\left([w, w][v, v]-[v, w]^{2}\right)}{2[u, u]^{1 / 2}[v, v]^{5 / 2}} .
$$

On account of the Cauchy-Schwarz inequality, the term in parenthesis is nonnegative and, hence, $f^{\prime \prime}\left(v_{u}, w\right) \leq 0$ for all $w \in \mathbb{H}_{T}(Q)$, so that $v=v_{u}$ indeed corresponds to the supremum. In summary, $G$ maps $u \in \mathbb{H}_{0}(Q)$ onto $[u, u]^{1 / 2}\left[v_{u}, v_{u}\right]^{-1 / 2} v_{u} \in \mathbb{H}_{T}(Q)$.

Consider now a function $u \in \mathbb{H}_{0}^{s}(q)$ extended to a distribution on $\mathbb{R}^{2}$. For the Fourier transform of $S u$ we obtain straightforwardly that $F S u=\hat{S} \hat{u}$ with $\hat{S}=\lambda^{2} \kappa^{4}-\omega^{2}$. Moreover, the identity $[v, w]=(S u, w)$ for all $w \in \mathbb{H}_{T}(Q)$ yields $\hat{H} \hat{v}=\hat{S} \hat{u}$, where $\hat{H}=\kappa^{4}+\omega^{2}$ represents the Fourier symbol pertaining to the inner product $[\cdot, \cdot]$. Hence, $F G u=\beta \hat{S} \hat{H}^{-1} \hat{u}$, the constant $\beta$ being determined by $[G u, G u]=[u, u]$.

\section{References}

[1] R.L. Bisplinghoff, H. Ashley, and R.L. Halfman. Aeroelasticity. Dover, New York, 1996.

[2] K. Bøhmer, P.W. Hemker, and H.J. Stetter. The defect correction approach. Computing, 5:1-32, 1984.

[3] A. Brandt. Multigrid techniques: 1984 guide with applications to fluid dynamics. Technical report, GMD, 1984.

[4] E.H. van Brummelen and R. de Borst. On the nonnormality of subiteration for a fluid-structure interaction problem. SIAM J. Sci. Comput., 27:599-621, 2005.

[5] E.H. van Brummelen, C. Michler, and R. de Borst. Interface-GMRES(R) acceleration of subiteration for fluid-structure-interaction problems. Technical Report DACS-05-001, Delft Aerospace Computational Science, 2005. Available at http://www.em.lr.tudelft.nl/ downloads/DACS-05-001.pdf.

[6] R. Dautray and J.L. Lions. Mathematical analysis and numerical methods for science and technology. Vol. 2. Functional and variational methods. Springer-Verlag, Berlin, 1988.

[7] E.H. Dowell. Aeroelasticity of Plates and Shells. Monographs and textbooks on mechanics of solids and fluids. Noordhoff, Leiden, 1975. 
[8] A. Ern and J.-L. Guermond. Theory and Practice of Finite Elements. Number 159 in Applied Mathematical Sciences. Springer-Verlag, New York, 2004.

[9] A. Greenbaum. Iterative Methods for Solving Linear Systems, volume 17 of Frontiers in Applied Mathematics. SIAM, Philadelphia, 1997.

[10] T.J.R. Hughes, G.R. Feijóo, L. Mazzei, and J.-B. Quincy. The variational multiscale method - a paradigm for computational mechanics. Comput. Methods Appl. Mech. Engrg., 166:3-24, 1998.

[11] T. Kato. Perturbation Theory for Linear Operators. Number 132 in Grundlehren der Mathematischen Wissenschaft. Springer, Berlin, corr. pr. 2nd ed. edition, 1984.

[12] J.L. Lions and E. Magenes. Non-Homogeous Boundary Value Problems and Applications I, volume 181 of Die Grundlehren der mathematischen Wissenschaften in Einzeldarstellungen mit besonderer Berücksichtigung der Anwendungsgebiete. Springer-Verlag, Berlin, 1972.

[13] W. McLean. Strongly Elliptic Systems and Boundary Integral Equations. Cambridge University Press, Cambridge, 2000.

[14] C. Michler. Efficient Numerical Methods for Fluid-Structure Interaction. PhD thesis, Delft University of Technology, 2005.

[15] C. Michler, E.H. van Brummelen, and R. de Borst. Error-amplification analysis of subiterationpreconditioned GMRES for fluid-structure interaction. Comput. Methods Appl. Mech. Engrg., 2005. (In Press).

[16] J.T. Oden and J.N. Reddy. An Introduction to the Mathematical Theory of Finite Elements. Pure and Applied Mathematics. John Wiley \& Sons, New York, 1974.

[17] L.N. Trefethen. Pseudospectra of linear operators. SIAM Rev., 39(3):383-406, 1997.

[18] G.B. Whitham. Linear and Nonlinear Waves. Pure and Applied Mathematics. Wiley, New York, 1974.

[19] E. Zeidler. Nonlinear Functional Analysis and its Applications II/B: Nonlinear Monotone Operators. Applied Mathematical Sciences. Springer, New York, 1990.

[20] E. Zeidler. Applied Functional Analysis: Applications to Mathematical Physics, volume 109 of Applied Mathematical Sciences. Springer, Berlin, 1995. 